\title{
AC transport and full-counting statistics of molecular junctions in the weak electron- vibration coupling regime
}

A. Ueda, Y. Utsumi, Y. Tokura, O. Entin-Wohlman, and A. Aharony

Citation: The Journal of Chemical Physics 146, 092313 (2017); doi: 10.1063/1.4973707

View online: http://dx.doi.org/10.1063/1.4973707

View Table of Contents: http://aip.scitation.org/toc/jcp/146/9

Published by the American Institute of Physics

\section{Articles you may be interested in}

Enhancing the conductivity of molecular electronic devices

The Journal of Chemical Physics 146, 092310092310 (2016); 10.1063/1.4972992

Field-induced inversion of resonant tunneling currents through single molecule junctions and the directional photo-electric effect

The Journal of Chemical Physics 146, 092314092314 (2017); 10.1063/1.4973891

Destructive quantum interference in electron transport: A reconciliation of the molecular orbital and the atomic orbital perspective

The Journal of Chemical Physics 146, 092308092308 (2016); 10.1063/1.4972572

Temperature dependent tunneling conductance of single molecule junctions

The Journal of Chemical Physics 146, 092311092311 (2017); 10.1063/1.4973318

Effects of vibrational anharmonicity on molecular electronic conduction and thermoelectric efficiency

The Journal of Chemical Physics 146, 092303092303 (2016); 10.1063/1.4965824

Electron transfer at thermally heterogeneous molecule-metal interfaces

The Journal of Chemical Physics 146, 092305092305 (2016); 10.1063/1.4971293

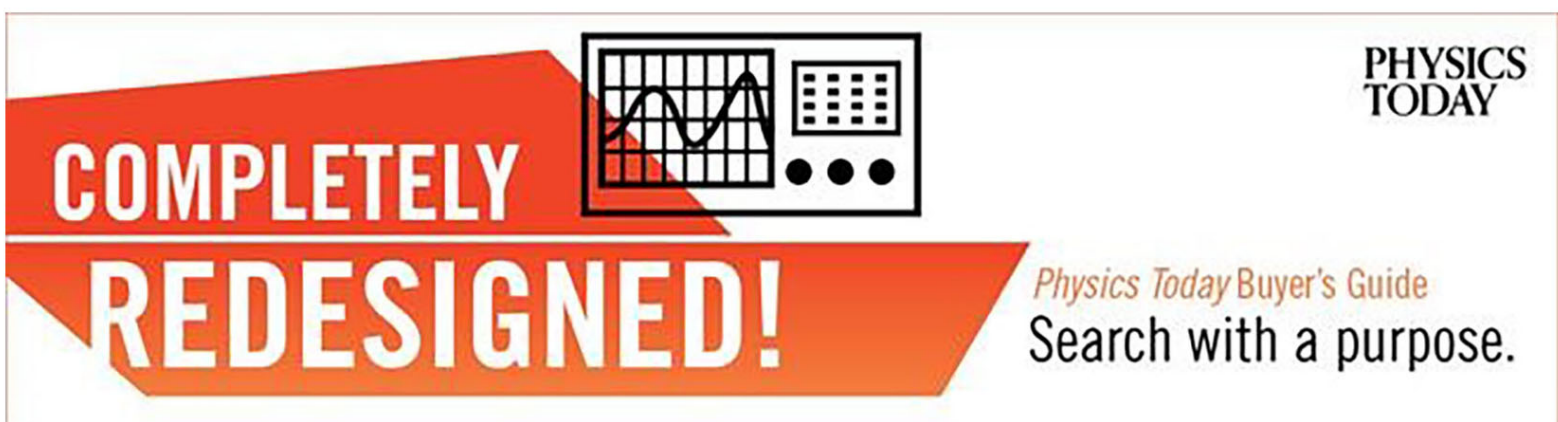




\title{
AC transport and full-counting statistics of molecular junctions in the weak electron-vibration coupling regime
}

\author{
A. Ueda, ${ }^{1}$ Y. Utsumi, ${ }^{2}$ Y. Tokura, ${ }^{3}$ O. Entin-Wohlman, ${ }^{4,5, a)}$ and A. Aharony ${ }^{4,5}$ \\ ${ }^{1}$ Faculty of Pure and Applied Sciences, Division of Applied Physics, University of Tsukuba, Tsukuba, \\ Ibaraki 305-8573, Japan \\ ${ }^{2}$ Department of Physics Engineering, Faculty of Engineering, Mie University, Tsu, Mie 514-8507, Japan \\ ${ }^{3}$ Faculty of Pure and Applied Sciences, Division of Physics, University of Tsukuba, Tsukuba 305-8573, Japan \\ ${ }^{4}$ Physics Department, Ben Gurion University, Beer Sheva 84105, Israel \\ ${ }^{5}$ Raymond and Beverly Sackler School of Physics and Astronomy, Tel Aviv University, Tel Aviv 69978, Israel
}

(Received 18 October 2016; accepted 21 December 2016; published online 18 January 2017)

\begin{abstract}
The coupling of the charge carriers passing through a molecule bridging two bulky conductors with local vibrational modes of the molecule gives rise to distinct features in the electronic transport properties on one hand and to nonequilibrium features in the vibrations' properties, e.g., their population, on the other. Here we explore theoretically a generic model for a molecular junction biased by an arbitrary dc voltage in the weak-coupling regime. We succinctly summarize parts of our past work related to the signature of the electron-vibration interaction on the full-counting statistics of the current fluctuations (i.e., the cumulant generating-function of the current correlations). In addition, we provide a novel account of the response to an ac field exerted on the junction (on top of the dc bias voltage); in particular, we study the nonequilibrium distribution and the displacement fluctuations of the vibrational modes. Remarkably, we find a behavior pattern that cannot be accounted for by classical forced oscillations. The calculations use the technique of nonequilibrium Green's functions and treat the electron-vibration coupling in perturbation theory, within the random-phase approximation when required. Published by AIP Publishing. [http://dx.doi.org/10.1063/1.4973707]
\end{abstract}

\section{INTRODUCTION}

Molecular junctions, which are metallic electrodes bridged by a single molecule (or a few molecules), are currently a subject of considerable interest due to their possible applications in molecular electronics. ${ }^{1}$ The particular feature of these setups, which distinguishes them from, e.g., quantum-dot or quantum-wire junctions, is the coupling between the motion of the molecule's vibrations, e.g., those of the center-of-mass, and the single-electron tunneling. Nanoelectro-mechanical vibrations were indeed detected in a single$\mathrm{C}_{60}$ transistor. $^{2}$ Early experiments, achieving almost a perfect transmission via a single molecule, were carried out on breakjunction devices bridged by $\mathrm{H}_{2} .{ }^{3}$ Conductances comparable to those of metallic atomic junctions were detected also for benzene molecules coupled to platinum leads. ${ }^{4}$ These are just a few examples of the huge body of experimental results concerning electric transport through molecular junctions. However, these devices have other attributes. Molecular junctions are particularly useful for studying electro-mechanical interactions in the quantum regime. When the bias voltage across a molecular junction exceeds the energy of a given mode of vibration, that mode can be excited, at low temperatures, by the electrons injected from the source electrode. This results in an additional contribution to the electric current. Whether this inelastic event increases or decreases the measured differential conductance is an intriguing question. At sufficiently strong

a) orawohlman@ gmail.com electron-vibration coupling, the current flow at low biases is found to be suppressed, a phenomenon termed the "FranckCondon blockade." ${ }^{5}$ On the other hand, a clear crossover between enhancement and reduction of the dc conductance was detected in shot-noise measurements, in a $\mathrm{H}_{2} \mathrm{O}$ molecular junction, ${ }^{6}$ and in gold nanowires. ${ }^{7}$ Interference effects on transport in molecular junctions have been studied both experimentally and theoretically, see, for example, Ref. 8. Beside electronic transport, other specifications of molecular junctions are being explored. ${ }^{9}$ Inelastic neutron tunneling spectroscopy ${ }^{10}$ and Raman response ${ }^{11}$ were used to study the molecular conformation and other characteristics of the junction itself. The electron-vibration coupling also induces renormalization, damping, ${ }^{12}$ and heating of the vibrational modes ${ }^{13}$ whose study could explain certain features in the Raman spectroscopy of OPV 3 junctions. ${ }^{14}$ Interestingly enough, the ability to measure the thermoelectric effects in molecular junctions provides a tool to determine the electronic structure of the molecule, for instance, by monitoring the Seebeck coefficient (for a recent review, see Ref. 15). Transport through molecular bridges coupled to metallic electrodes has been also exploited to investigate electronic correlations, e.g., bias-induced charging of the junction ${ }^{16}$ or the Kondo effect; ${ }^{17}$ see, however, Ref. 18 for a different interpretation for the latter observation.

The theoretical analysis of transport through molecular junctions has been carried out by a vast variety of methods. These include ab initio computations (see, e.g., Refs. 19-22), mixed quasi-classical and semiclassical approaches (see, e.g., Refs. 23 and 24), calculations based on the scattering theory, ${ }^{25-27}$ constructions of quantum master equations, ${ }^{28}$ 
using real-time path-integrals combined with Monte Carlo computations, ${ }^{29}$ and more.

In this paper, we consider the effect of the electronvibration coupling on transport properties at an arbitrary bias voltage, i.e., when transport is beyond the linear-response regime. The coupling of the vibrations with the charge carriers naturally involves also inelastic processes. At very low temperatures, as considered in this paper, real inelastic scattering events are feasible when the bias voltage exceeds the threshold of the vibrational modes' energy. One therefore expects unique features at bias voltages around this energy. The application of an additional ac field as considered below gives rise to an interplay between the ac frequency and the frequencies of the vibrational modes. The focus of our paper is the study of the dynamics of the charge carriers and that of the vibrations of the molecule over a wide range of bias voltages, vibrational frequencies, and ac frequencies.

Under these circumstances, a suitable method to use is that of the nonequilibrium Green's functions, i.e., the Keldysh technique. ${ }^{30}$ We apply this technique to the ubiquitous simple model for molecular junctions, which replaces the molecule by a quantum dot with a single localized level attached to two electronic reservoirs. Electrons residing on the level exchange energy with Einstein vibrations (or optical phonons), of frequency $\omega_{0}$, resulting from oscillations of the junction, as represented by the localized level. Even for a weak electronphonon coupling, this model, which has been pursued for more than a decade, produces intriguing features in the transport properties. ${ }^{31-33}$ As in our previous works on this topic, ${ }^{33-38}$ we confine ourselves to this regime, treating the electronvibration interaction in the lowest possible order in the coupling energy. ${ }^{32,33,39-42}$ Note, however, that this procedure is rather delicate and care must be taken in exploiting it (see, e.g., the discussion in Sec. III). The limit where the vibrations are strongly coupled to the charge carriers, ${ }^{43-47}$ and the effect of electron-electron correlations, ${ }^{48-51}$ is beyond the scope of this paper.

While considerable theoretical effort has been devoted to the study of dc transport, less attention has been paid to the response of molecular junctions to a frequency-dependent electric field. The ac conductance for tunneling through an arbitrary interacting quantum dot was analyzed in Ref. 52, and polaronic effects were considered in Ref. 53, assuming that the vibrational modes are equilibrated on a time scale shorter than the transit time of the electrons through the junction. We focus on the situation where the vibrational modes are equilibrated via their interaction with the charge carriers; in particular, we explore the effect on the full counting statistics (FCS), and the modifications introduced by an ac field in the nonequilibrium distribution of the vibrational modes, together with its effect on the oscillations of the center of mass of the molecule.

Our paper is organized as follows. In Sec. II A we describe the model used for the calculations. To set the stage for the discussion of the ac current in the presence of arbitrary dc voltages, in linear response to an ac field, we review in Sec. II B certain properties of the dc current at finite voltages. Section II C contains a detailed analysis of the ac response of the junction; particular attention is paid to the dependence of the response coefficient on the ac frequency. This part of the calculation involves the consideration of various diagrams, whose individual contributions to the ac transport coefficient are not easy to anticipate. We list in the Appendix these diagrams, their detailed expressions, and display the plots of their separate contributions to the ac response. The investigation of the effect of an ac field on the dynamics of the junction is continued in Sec. II D, where we study two correlation functions of the vibrations: the first is related to the nonequilibrium distribution of the vibrations, and the other to the fluctuation in the displacement of the harmonic oscillator representing the junction. The dependence of the two quantities on the ac frequency is analyzed. In particular, we find that the phase delay of the fluctuation shows a structure at two specific values of that frequency: one which can be explained by considering a classical driven oscillator, and another which cannot; it stems from twovibration scattering by the charge carriers and thus exemplifies a quantum electron-mechanical effect. Section III reviews our recent results for the cumulant generating-function (CGF) of our model and dwells in particular on its modifications due to the electron-vibration coupling and the nonequilibrium distribution of the vibrations. An intriguing relation between the full counting statistics and the theory of thermodynamic phase transitions is pointed out. Section IV summarizes briefly our work.

\section{ELECTRIC CURRENT AND VIBRATIONAL MODES DYNAMICS}

\section{A. The model Hamiltonian and the electric current}

The model we use is depicted in Fig. 1: a localized electronic level, of energy $\epsilon_{0}$, is coupled to two electronic electrodes, which are held at two different chemical potentials, $\mu_{L}+\delta \mu_{L}(t)$ and $\mu_{R}+\delta \mu_{R}(t)$. An ac field of frequency $\omega_{\text {ac }}$ applied to the junction is represented by a periodic timedependence of the chemical potentials, $\delta \mu_{L(R)}(t)$. Specifically we choose

$$
\begin{aligned}
& \mu_{L}+\delta \mu_{L}(t)=\mu+e V / 2+\delta \mu_{L} \cos \left(\omega_{\mathrm{ac}} t\right), \\
& \mu_{R}+\delta \mu_{R}(t)=\mu-e V / 2+\delta \mu_{R} \cos \left(\omega_{\mathrm{ac}} t\right),
\end{aligned}
$$

where $V$ is the bias voltage, and $\mu$ is the common chemical potential of the electrodes. An electron on the level is coupled to local Einstein vibrations; this coupling induces fluctuations in the level energy. ${ }^{31-33,54,55}$ The model Hamiltonian is

$$
\mathcal{H}=\mathcal{H}_{\text {lead }}+\mathcal{H}_{\text {mol }}+\mathcal{H}_{\text {ph }}+\mathcal{H}_{\text {tun }} .
$$

The two electronic electrodes (assumed to be identical except being kept at different chemical potentials) are represented by free-electron gases,

$$
\begin{aligned}
\mathcal{H}_{\text {lead }}= & \sum_{k}\left(\epsilon_{k}-\mu-e V / 2\right) c_{k}^{\dagger} c_{k} \\
& +\sum_{p}\left(\epsilon_{p}-\mu+e V / 2\right) c_{p}^{\dagger} c_{p},
\end{aligned}
$$

where $c_{k(p)}^{\dagger}$ and $c_{k(p)}$ denote the creation and annihilation operators of an electron of momentum $k(p)$ and energy $\epsilon_{k(p)}$ in the left (right) electrode, respectively. The Hamiltonian of the localized level reads

$$
\mathcal{H}_{\text {mol }}=\left[\epsilon_{0}+\gamma\left(b+b^{\dagger}\right)\right] c_{0}^{\dagger} c_{0},
$$




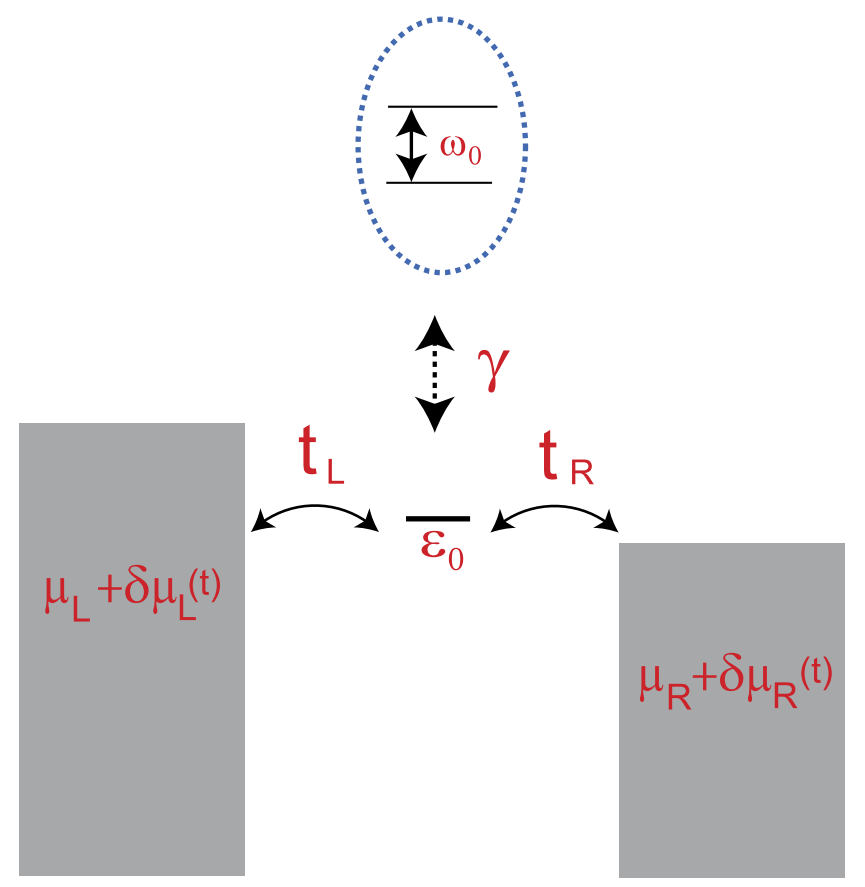

FIG. 1. Illustration of the model used in the calculation. An electronic localized level, of energy $\epsilon_{0}$, is coupled to two bulky electronic reservoirs, held at two different chemical potentials, $\mu_{L(R)}+\delta \mu_{L(R)}(t)$ for the left (right) electrode. The charge carriers exchange energy with Einstein vibrations of frequency $\omega_{0}$; the coupling energy of the electrons with the vibrations is denoted $\gamma$. The difference $\mu_{L}-\mu_{R}=e V$ is the bias voltage multiplied by the unit of charge; $\delta \mu_{L(R)}(t)$ are monochromatic ac fields of frequency $\omega_{\mathrm{ac}}$ applied to the junction. These are treated in the linear-response approximation.

with the creation and annihilation operators, $c_{0}^{\dagger}$ and $c_{0}$, respectively, for an electron on the localized level. The second term in the square brackets is the (linear) electron-vibration coupling: the creation and annihilation operators of the Einstein vibrations are $b^{\dagger}$ and $b$, respectively, and the electronvibration coupling energy is $\gamma$. The vibrational modes obey the Hamiltonian

$$
\mathcal{H}_{\mathrm{ph}}=\omega_{0} b^{\dagger} b
$$

(We use units in which $\hbar=1$.) Our calculations are carried out in second-order perturbation theory in the electron-vibration coupling, i.e., we keep terms up to order $\gamma^{2}$. However, in the absence of the ac field, this approximation is not sufficient for the determination of the vibrations' population [see the discussions following Eq. (30) and in Sec. III].

The tunneling Hamiltonian connecting the localized level with the left (right) electrode is specified by the tunneling amplitude $t_{L(R)}$ and is written in the form

$$
\mathcal{H}_{\text {tun }, \pm}=\sum_{k} t_{L} e^{ \pm i \lambda_{L}} c_{k}^{\dagger} c_{0}+\sum_{p} t_{R} e^{ \pm i \lambda_{R}} c_{p}^{\dagger} c_{0}+\text { H.c. }
$$

Here, $\lambda_{L(R)}$ are the counting fields. ${ }^{37,56}$ These are introduced to facilitate the calculation of the full-counting statistics (Sec. III). In the long-time limit, the cumulant generatingfunction depends only on the difference of the two, i.e., on

$$
\lambda=\lambda_{L}-\lambda_{R}
$$

As $\lambda_{L}+\lambda_{R}$ counts the number of electrons flowing into the localized level, the fact that the cumulant generating-function depends solely on $\lambda$ implies charge conservation. For the calculation of the response of the system to the chemical potentials, we set $\lambda_{L}=\lambda_{R}=0$.

The average (time-dependent) current emerging from the left electrode is

$$
\begin{aligned}
I_{L}(t) & =-e\left\langle\frac{d}{d t} \sum_{k} c_{k}^{\dagger} c_{k}\right\rangle \\
& =e \operatorname{Re}\left(\int d t^{\prime} \sum_{k}\left|t_{L}\right|^{2}\left[G_{00}^{r}\left(t, t^{\prime}\right) g_{k}^{<}\left(t^{\prime}, t\right)+G_{00}^{<}\left(t, t^{\prime}\right) g_{k}^{a}\left(t^{\prime}-t\right)-g_{k}^{r}\left(t-t^{\prime}\right) G_{00}^{<}\left(t^{\prime}, t\right)-g_{k}^{<}\left(t, t^{\prime}\right) G_{00}^{a}\left(t^{\prime}, t\right)\right]\right)
\end{aligned}
$$

The current emerging from the right electrode is obtained from Eq. (8) by the replacements $L \rightarrow R$ and $k \rightarrow p$. Here, $G_{00}$ is the Green's function on the localized level in the presence of the coupling to the electrodes, the ac fields $\delta \boldsymbol{\mu}_{L(R)}$, and the electron-vibration interaction. The retarded and the advanced electron Green's functions are

$$
G_{00}^{r / a}\left(t, t^{\prime}\right)=\mp i \Theta\left( \pm t \mp t^{\prime}\right)\left\langle\left\{c_{0}(t), c_{0}^{\dagger}\left(t^{\prime}\right)\right\}\right\rangle,
$$

and the Keldysh lesser Green's function ${ }^{30}$ is

$$
G_{00}^{<}\left(t, t^{\prime}\right)=i\left\langle c_{0}^{\dagger}\left(t^{\prime}\right) c_{0}(t)\right\rangle
$$

The Green's functions on the decoupled electrodes are denoted by the lowercase $g_{k(p)}$, with

$$
g_{k(p)}^{r / a}\left(t, t^{\prime}\right)=\mp i \Theta\left( \pm t \mp t^{\prime}\right)\left\langle\left\{c_{k(p)}(t), c_{k(p)}^{\dagger}\left(t^{\prime}\right)\right\}\right\rangle
$$

and

$$
g_{k(p)}^{<}\left(t, t^{\prime}\right)=i\left\langle c_{k(p)}^{\dagger}\left(t^{\prime}\right) c_{k(p)}(t)\right\rangle
$$

We consider the response of the junction to the ac fields in linear response. That is, while the bias voltage is arbitrary, $\delta \mu_{L(R)}$ [Eq. (1)] are assumed to be small. To this end, we use the expansion

$$
\begin{aligned}
\sum_{k(p)} g_{k(p)}^{<}\left(t, t^{\prime}\right)= & i v \int d \epsilon_{k(p)} f_{L(R)}\left(\epsilon_{k(p)}\right) e^{-i \epsilon_{k(p)}\left(t-t^{\prime}\right)} \\
& \times \exp \left[i \int_{t^{\prime}}^{t} d t_{1} \delta \mu_{L(R)} e^{i \omega_{a c} t_{1}}\right]
\end{aligned}
$$

where

$$
f_{L(R)}(\omega)=\left[e^{\beta(\omega-\mu \mp e V / 2)}+1\right]^{-1}
$$


is the Fermi distribution function of the electrons in the left (right) electrode, and $\beta=1 /\left(k_{B} T\right)$ is the inverse temperature. In Eq. (13), $v$ is the density of states of the electron gases in the electrodes, at the common chemical potential. [Note that $\delta \mu_{L(R)} \cos \left(\omega_{\mathrm{ac}} t\right)$ is replaced in the calculations by $\left.\delta \mu_{L(R)} \exp \left(i \omega_{\mathrm{ac}} t\right) .{ }^{35}\right]$ By expanding Eq. (13) to linear order in the chemical potentials, we find

$$
I_{L}(t) \approx I_{L}+C_{L L}\left(\omega_{\mathrm{ac}}\right) e^{i \omega_{\mathrm{ac}} t} \delta \mu_{L}+C_{L R}\left(\omega_{\mathrm{ac}}\right) e^{i \omega_{\mathrm{ac}} t} \delta \mu_{R},
$$

where $I_{L}$ is the dc current flowing from the left electrode. The other terms on the right-hand side of Eq. (15) describe the ac current emerging from the left electrode in linear response (with respect to $\delta \mu_{L}$ and $\delta \mu_{R}$ ).

We adopt the definition

$$
I(t)=\left[I_{L}(t)-I_{R}(t)\right] / 2
$$

for the current flowing through the junction. In Sec. II B we examine the dc current; Sec. II C analyzes the response to the ac field, assuming for concreteness that $\delta \mu_{L}+\delta \mu_{R}=0$, and confining the discussion to a spatially symmetric junction. With these assumptions, the response to the ac field is given by a single coefficient

$$
C\left(\omega_{\mathrm{ac}}\right)=\left[C_{L}\left(\omega_{\mathrm{ac}}\right)+C_{R}\left(\omega_{\mathrm{ac}}\right)\right] / 2 .
$$

(Since part of the coefficient $C_{L L(R R)}$ is cancelled against $C_{L R(R L)}$, the notations are modified. ${ }^{35}$ ) We remind the reader that while the ac field is treated in linear response, the bias voltage $V$ is arbitrary.

\section{B. The dc current}

In the absence of the ac field (but in the presence of a constant bias voltage), the dc current through the junction can be written in the form (see, for instance, Ref. 33)

$$
\begin{aligned}
I & =\left(I_{L}-I_{R}\right) / 2 \\
& =e \frac{\Gamma_{L} \Gamma_{R}}{\Gamma} \int \frac{d \omega}{2 \pi} \operatorname{Im} G_{00}^{a}(\omega)\left[f_{L}(\omega)-f_{R}(\omega)\right] .
\end{aligned}
$$

Due to the coupling with the leads, the localized level becomes a resonance, whose width is $\Gamma$,

$$
\Gamma=\Gamma_{L}+\Gamma_{R}, \quad \Gamma_{L(R)}=2 \pi v\left|t_{L(R)}\right|^{2},
$$

where $\Gamma_{L}\left(\Gamma_{R}\right)$ is the partial width arising from the coupling to left (right) electrode. These partial widths determine the transmission $\mathcal{T}$ of the junction at the average chemical potential of the leads,

$$
\mathcal{T}=\frac{\Gamma_{L} \Gamma_{R}}{\left(\mu-\epsilon_{0}\right)^{2}+(\Gamma / 2)^{2}} .
$$

This derivation assumes that the tunnel coupling and the electronic density of states can be replaced by their values at the average chemical potential of the leads, that is, $t_{L}$ and $t_{R}$ are not modified by the coupling with the vibrations. An alternative treatment would be to first carry out the small-polaron transformation, which transforms $t_{L}$ and $t_{R}$ into functions of the operators $b$ and $b^{\dagger}$. Here we follow a straightforward secondorder calculation in $\gamma$. To order $\mathcal{O}\left(\gamma^{2}\right)$, the two approaches are identical. The Green's function $G_{00}^{a}(\omega)$ is the Fourier transform of $G_{00}^{a}\left(t-t^{\prime}\right)$, Eq. (9) [note that at steady state the functions in
Eq. (9) depend only on the time difference]; to second order in the electron-vibration coupling $\gamma$, it reads ${ }^{33}$

$$
G_{00}^{r / a}(\omega)=\left[\omega-\epsilon_{0} \pm i \Gamma / 2-\Sigma_{\mathrm{Har}}^{r / a}-\Sigma_{\mathrm{Ex}}^{r / a}(\omega)\right]^{-1} .
$$

Here $\Sigma_{\text {Har }}$ and $\Sigma_{\mathrm{Ex}}(\omega)$ constitute the self-energy due to that interaction. The Hartree term of the interaction gives rise to a frequency-independent self energy,

$$
\Sigma_{\mathrm{Har}}^{r / a}=-i \gamma^{2} \int \frac{d \omega}{2 \pi} G_{00}^{(0)<}(\omega) D^{r / a}(0),
$$

and the exchange term is

$$
\begin{aligned}
\Sigma_{\mathrm{Ex}}^{r / a}(\omega)= & i \gamma^{2} \int \frac{d \omega^{\prime}}{2 \pi}\left(G_{00}^{(0)<}\left(\omega-\omega^{\prime}\right) D^{r / a}\left(\omega^{\prime}\right)\right. \\
& \left.+G_{00}^{(0) r / a}\left(\omega-\omega^{\prime}\right)\left[D^{<}\left(\omega^{\prime}\right) \pm D^{r / a}\left(\omega^{\prime}\right)\right]\right) .
\end{aligned}
$$

The electron Green's functions appearing in Eqs. (22) and (23) are zeroth-order in the electron-vibration coupling, as these self-energies are proportional to $\gamma^{2}$. The expressions for these Green's functions are

$$
G_{00}^{(0) r / a}(\omega)=\left[\omega-\epsilon_{0} \pm i \Gamma / 2\right]^{-1}
$$

and

$G_{00}^{(0)<}(\omega)=i\left[\Gamma_{L} f_{L}(\omega)+\Gamma_{R} f_{R}(\omega)\right] G_{00}^{(0) a}(\omega) G_{00}^{(0) r}(\omega)$.

[The greater Green's function is obtained upon replacing $f_{L(R)}$ by $\left.\left.f_{L(R}\right)-1.\right]$ The Hartree term in the self energy is ignored hereafter, as it just produces a shift in the localized-level energy.

The expressions for the self-energies include also the vibration Green functions, $D$,

$$
\begin{aligned}
& D^{r / a}\left(t, t^{\prime}\right)=\mp i \Theta\left( \pm t \mp t^{\prime}\right)\left\langle\left[b(t)+b^{\dagger}(t), b\left(t^{\prime}\right)+b^{\dagger}\left(t^{\prime}\right)\right]\right\rangle, \\
& D^{<}\left(t, t^{\prime}\right)=-i\left\langle\left[b\left(t^{\prime}\right)+b^{\dagger}\left(t^{\prime}\right)\right]\left[b(t)+b^{\dagger}(t)\right]\right\rangle .
\end{aligned}
$$

At steady state, the Fourier transforms of the vibration Green's functions are

$$
D^{r / a}(\omega)=\frac{2 \omega_{0}}{(\omega \pm i \eta)^{2}-\omega_{0}^{2}+2 \omega_{0} \Pi^{r / a}(\omega)}
$$

for the retarded and advanced functions and

$$
D^{<}(\omega)=D^{r}(\omega) \Pi^{<}(\omega) D^{a}(\omega)
$$

for the lesser Green's function. In Eqs. (27) and (28), $\eta$ characterizes the approach of the vibrations' distribution to its equilibrium value (see below), $\Pi$ is the electron-hole propagator,

$$
\begin{aligned}
\Pi^{r / a}(\omega)= & -i \gamma^{2} \int \frac{d \omega^{\prime}}{2 \pi}\left[G_{00}^{r / a}\left(\omega^{\prime}\right) G_{00}^{<}\left(\omega^{\prime}-\omega\right)\right. \\
& \left.+G_{00}^{<}\left(\omega^{\prime}\right) G_{00}^{a / r}\left(\omega^{\prime}-\omega\right)\right]
\end{aligned}
$$

and

$$
\Pi^{<}(\omega)=-i \gamma^{2} \int \frac{d \omega^{\prime}}{2 \pi} G_{00}^{<}\left(\omega^{\prime}\right) G_{00}^{>}\left(\omega^{\prime}-\omega\right),
$$

where $G_{00}^{>}$is the greater Keldysh function. ${ }^{30}$ Since the electron-hole propagator is proportional to $\gamma^{2}$, the Green's functions $G_{00}$ in Eqs. (29) and (30) are those of the interactionfree junction, Eqs. (24) and (25).

There is a subtle feature in this model, which distinguishes between the equilibration processes of the electrons and of 
the vibrational modes. While the electrodes serve as thermal baths for the electrons, where they acquire the Fermi distribution (different for the two electrodes), the model, as described in Sec. II A, does not specify the relaxation of the vibrational modes. In other words, whether or not the vibrations can equilibrate in a time much shorter than the transit time of an electron through the junction affects significantly the dynamics. When it is plausible to assume that the distribution of the vibrational modes is determined by their coupling to a phonon bath (e.g., the substrate on which the junction is lying) and is given by the Bose-Einstein distribution, $\eta \gg \operatorname{Im}\left[\Pi^{a}(\omega)\right]$. In contrast, in the other extreme case, $\operatorname{Im}[\Pi]$ dominates, and the vibrational modes' distribution is governed by the coupling to the charge carriers. $^{57}$

Figure 2 displays the dependence of the differential conductance, $G$, on the bias voltage and on the other parameters of the junction and illustrates the difference between the two possibilities discussed above, i.e., $\eta \ll \operatorname{Im}\left[\Pi^{a}(\omega)\right]$ and $\eta>>\operatorname{Im}\left[\Pi^{a}(\omega)\right]$. Figure 2(a) shows the conductance of a junction tightly bound to the leads (the resonance width $\Gamma$ is relatively large) and hence the dwell time of the electrons is rather short. Then, at relatively low values of the bare transmission, $\mathcal{T}=0.3$, there is no discernible modification in the conductance which is about the same for nonequilibrium
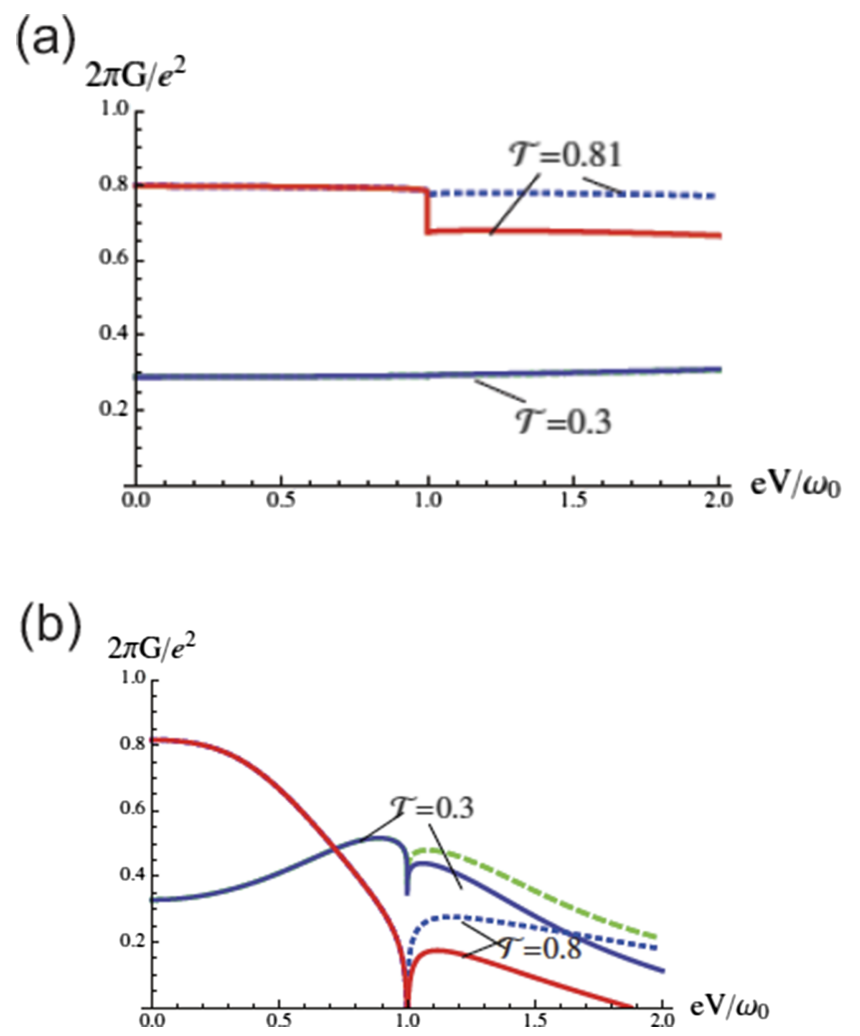

FIG. 2. The zero-temperature differential conductance $G$ (scaled by the quantum unit of the conductance) as a function of the bias voltage (scaled by $\omega_{0} / e$ ), for two values of the junction's transmission $\mathcal{T}$ (marked in the figure). The full lines [for $\mathcal{T}=0.81$ (red) and $\mathcal{T}=0.3$ (dark blue)] are the conductance when the vibrations' population is determined by the coupling to the charge carriers, and the dotted curves [for $\mathcal{T}=0.81$ (blue) and $\mathcal{T}=0.3$ (green)] are for a Bose-Einstein population. The coupling energy $\gamma$ is $\omega_{0} / 2$ and $\Gamma=6 \omega_{0}$ (a) and $\omega_{0} / 6$ (b). Adapted with permission from Entin-Wohlman et al., Phys. Rev. B 81, 113408 (2010). Copyright 2010 The American Physical Society. vibrational modes' population and for the equilibrium one. Figure 2(b) depicts the situation when the resonance width is relatively small. There, for higher values of the bare transmission, $\mathcal{T}=0.81$, the step-down feature of the conductance at threshold for inelastic tunneling, $e V=\omega_{0}$, is enhanced for nonequilibrium vibrations as compared to the equilibrated ones. Hence, when the bare transmission of the junction is high, the conductance in the presence of nonequilibrium population is lower than the one pertaining to the case of equilibrated vibrations. For low bare transmissions, the difference is rather small. One notes that the logarithmic singularity associated with the real part of the self-energy at the channel opening ${ }^{33}$ is manifested also when the vibrations are out of equilibrium, for high enough values of the bare transmission of the junction. The fact that the changes in the differential conductance are more pronounced for the larger values of $\omega_{0} / \Gamma$ is connected with the actual value of the population. The electrons pump more and more excitations into the higher vibrational states as the bias voltage increases and this pumping is more effective as the dwell time exceeds considerably the response time of the oscillator.

\section{The ac current}

In our previous work, ${ }^{35}$ we have found that the response of the charge carriers to a frequency-dependent field, when the bias voltage vanishes, can be enhanced (suppressed) by the coupling to the vibrations when the localized level lies below (above) the common chemical potential of the electrodes; this behavior was attributed to the Franck-Condon blockade due to the Hartree term, Eq. (22). It was also found that the vertex corrections of that interaction induce an additional peak structure in the response, which could be modified by tuning the width of the resonance, i.e., by varying $\Gamma_{L(R)}$. This additional peak disappeared in a spatially symmetric junction, for which $\Gamma_{L}=\Gamma_{R}$. In a more recent work, ${ }^{38}$ we have included also the effect of a finite bias voltage, assuming that the vibrations are equilibrated by their coupling to both a phonon bath [as described by the $\eta$, see the discussion following Eq. (30)] and the charge carriers (a possibility that exists at very low temperatures when $V \neq 0$ ). That analysis pertained to a perfect junction for which $\mathcal{T}=1$ [see Eq. (20)]. It was found that the response can be enhanced by the coupling to the vibrations for sufficiently large values of the bias voltage. In addition, $C\left(\omega_{\mathrm{ac}}\right)$ developed a sharp feature at $\omega_{\mathrm{ac}}=2 \omega_{0}$; we discuss this structure below. Here we extend that study for non-perfect junctions, i.e., for $\mathcal{T}<1$.

The detailed expressions for the Fourier transform of the transport coefficient $C\left(\omega_{\mathrm{ac}}\right)$ are given in the Appendix, see also Ref. 38. They are based on the expansion of the electron Green's function, in the absence of the coupling with vibrations, to linear order in the ac amplitude $\delta \mu=\delta \mu_{L}=-\delta \mu_{R}$,

$$
G_{00}^{(\mathrm{nint})<}\left(t, t^{\prime}\right)=G_{00}^{(0)<}\left(t-t^{\prime}\right)+G_{00}^{(\mathrm{ac})<}\left(t, t^{\prime}\right),
$$

where the Fourier transform of the first term on the right-hand side is given in Eq. (25), and 


$$
\begin{aligned}
G_{00}^{(\mathrm{ac})(0)<}\left(\omega_{\mathrm{ac}}, \omega\right)= & \frac{i \Gamma \delta \mu}{\omega_{\mathrm{ac}}} G_{00}^{(0) r}\left(\omega-\omega_{\mathrm{ac}}\right) G_{00}^{(0) a}(\omega) \\
& \times\left[f_{L}\left(\omega-\omega_{\mathrm{ac}}\right)-f_{L}(\omega)\right. \\
& \left.-f_{R}\left(\omega-\omega_{\mathrm{ac}}\right)+f_{R}(\omega)\right] .
\end{aligned}
$$

The Green's function Eq. (32) is written for a spatially symmetric junction, $\Gamma_{L}=\Gamma_{R}=\Gamma / 2$ [see Eq. (19)]. The relevant diagrams (whose expressions are given in the Appendix) are depicted in Fig. 8.

The transport coefficient $C\left(\omega_{\mathrm{ac}}\right)$, in the presence and in the absence of the electron-vibration interaction, is plotted in Fig. 3 as a function of $\omega_{\mathrm{ac}} / \omega_{0}$. Panels (a) and (b) there show it for a high-transparency junction, $\mathcal{T}=0.8$, for $e V=0.5 \omega_{0}$ and $e V=2 \omega_{0}$, respectively. The other parameters are $\mu-\epsilon_{0}=0.125 \omega_{0}, \Gamma=0.5 \omega_{0}$ for the upper pair of curves, and $\mu-\epsilon_{0}=0.5 \omega_{0}, \Gamma=2 \omega_{0}$ for the lower pair. The coupling of the charge carriers to the vibrations suppresses $C\left(\omega_{\mathrm{ac}}\right)$ for both pairs of parameters when the bias voltage is low, $e V=0.5 \omega_{0}$. The coupling to the vibrations has almost no effect on $C\left(\omega_{\mathrm{ac}}\right)$ as the ac frequency exceeds the vibrations' frequency; one might say that the vibrations cannot "catch up" there. For the higher bias voltage, $e V=2 \omega_{0}$, the coupling with the vibrations causes $C\left(\omega_{\mathrm{ac}}\right)$ to diminish for the large resonance width, $\Gamma=2 \omega_{0}$, but enhances it for the smaller value of $\Gamma\left(=0.5 \omega_{0}\right)$ when $\omega_{\mathrm{ac}}<\omega_{0}$. Panels (c) and (d) in Fig. 3 display $C\left(\omega_{\mathrm{ac}}\right)$ for a low-transmission junction, $\mathcal{T}=0.2$. Here, $\mu-\epsilon_{0}=0.5 \omega_{0}, \Gamma=0.5 \omega_{0}$ for one pair of curves, and $\mu-\epsilon_{0}=2 \omega_{0}, \Gamma=2 \omega_{0}$ for the other. It is seen that the coupling to the vibrations enhances $C\left(\omega_{\mathrm{ac}}\right)$ at small ac frequencies for $e V=0.5 \omega_{0}$ [Fig. 3(c)], but decreases it for the higher ones: $0.8 \omega_{0} \lesssim \omega_{\mathrm{ac}}$ for $\Gamma=0.5 \omega_{0}$ and $2.3 \omega_{0} \lesssim \omega_{\mathrm{ac}}$ for $\Gamma=2 \omega_{0}$. At $e V=2 \omega_{0}$ [Fig. 3(d)] $C\left(\omega_{\mathrm{ac}}\right)$ diminishes when $\omega_{\mathrm{ac}} \lesssim 0.5 \omega_{0}$ for both values of $\Gamma$, but increases for $\omega_{\mathrm{ac}} \sim 0.7 \omega_{0}$ and $\Gamma=0.5 \omega_{0}$, and for $\omega_{\mathrm{ac}} \sim \omega_{0}$ and $\Gamma=2 \omega_{0}$, respectively. Again we find that $C\left(\omega_{\mathrm{ac}}\right)$ tends to decrease gradually at high ac frequencies. The Appendix examines the contribution of each diagram separately to $C\left(\omega_{\mathrm{ac}}\right)$, see Figs. 9 and 10 .

\section{Features of the vibrations under an ac field}

Within the framework of the Hamiltonian Eq. (2), the dynamics of the vibrational modes is brought about via their coupling with the charge carriers; they are affected by the ac field also only through that interaction. Technically, the dynamics of the vibrations is described by the particle-hole propagator, see the diagrams in Fig. 8. Here we focus our attention on the ac field-induced modifications of two quantities. The first comes from the correlation $d^{<}\left(t, t^{\prime}\right)=-i\left\langle b^{\dagger}\left(t^{\prime}\right) b(t)\right\rangle$, the second from the usual vibration Green's functions, given in Eq. (26).

Formally, the distribution of the vibrational modes is given by the equal-time correlation

$$
N(t)=i d^{<}(t, t), \quad d^{<}(t, t)=-i\left\langle b^{\dagger}(t) b(t)\right\rangle .
$$

The Green's function $d^{<}(t, t)$ is derived by solving its equation of motion, treating the electron-vibration coupling $\gamma$ up to the second order. The definition (33) does not coincide with the one given in Refs. 13 and 40, which uses the equal-time Green's function $D$ [Eq. (26)], that is, $N(t)=\left[-1+i D^{<}(t, t)\right] / 2$. The reason is the presence of the ac field which causes $\left\langle\left[b^{\dagger}(t)\right]^{2}\right\rangle$ and $\left\langle[b(t)]^{2}\right\rangle$ to be nonzero. The equal-time conventional vibration Green's function $D^{<}(t, t)$ [see Eq. (26)] yields the fluctuation of the displacement of the harmonic oscillator representing the junction, $(\Delta X)^{2}$, as the coordinate operator $\hat{X}$ along the junction (assumed to lie along the $\mathrm{x}$ direction) is given by

$$
\hat{X} \sqrt{2 \omega_{0}}=\left(b+b^{\dagger}\right)
$$

Hence

$$
i D^{<}(t, t)=2 \omega_{0}[\Delta X(t)]^{2} .
$$
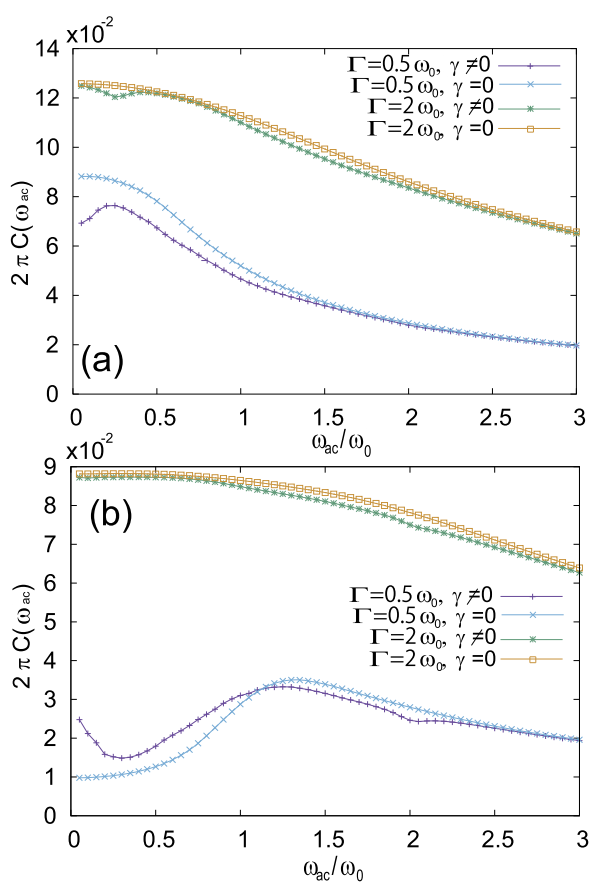
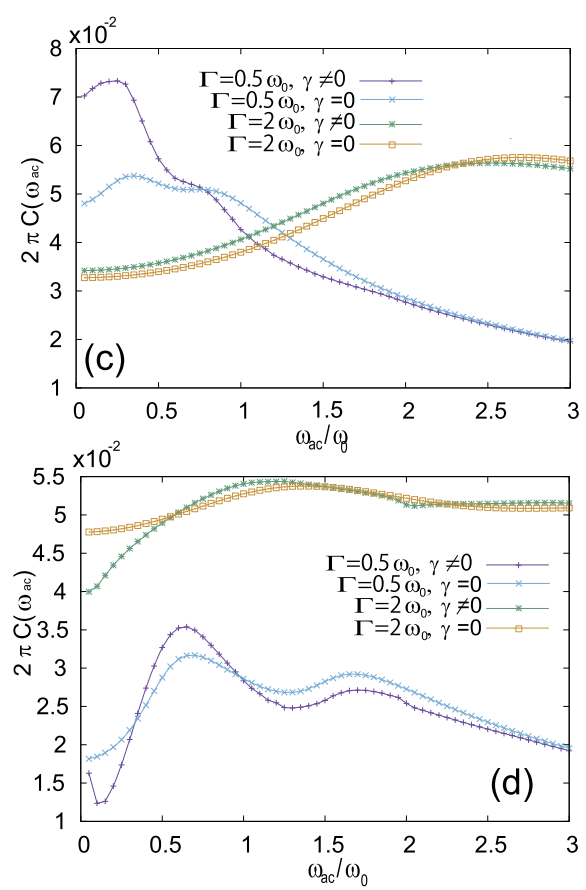

FIG. 3. The zero-temperature transport coefficient $C\left(\omega_{a c}\right)$ [Eq. (17)] as a function of the ac-field frequency, measured in units of $\omega_{0}$, for various values of the junction transmission $\mathcal{T}$ and the bias voltage $V$. (a) $\mathcal{T}=0.8$, $e V=0.5 \omega_{0}$; (b) $\mathcal{T}=0.2, e V=0.5 \omega_{0}$; (c) $\mathcal{T}=0.8, \quad e V=2 \omega_{0} ;$ (d) $\mathcal{T}=0.2$, $e V=2 \omega_{0}$. There are two pairs of curves in each panel. The members of each pair correspond to the same value of the resonance width (either $\Gamma=0.5 \omega_{0}$ or $\left.\Gamma=2 \omega_{0}\right)$; one of them is plotted for zero electron-vibration coupling, and the other for $\gamma=0.5 \omega_{0}$. 
We expand both quantities, Eqs. (33) and (35), to linear order in the ac chemical potentials, to obtain (for the Fourier transforms at the ac frequency)

$$
N\left(\omega_{\mathrm{ac}}\right)=N^{(0)}+N_{L}^{(1)}\left(\omega_{\mathrm{ac}}\right) \delta \mu_{L}+N_{R}^{(1)}\left(\omega_{\mathrm{ac}}\right) \delta \mu_{R}
$$

and

$$
\begin{aligned}
{\left[\Delta X\left(\omega_{\mathrm{ac}}\right)\right]^{2}=} & {\left[\Delta X^{(0)}\left(\omega_{\mathrm{ac}}\right)\right]^{2}+\left[\Delta X_{L}^{(1)}\left(\omega_{\mathrm{ac}}\right)\right]^{2} \delta \mu_{L} } \\
& +\left[\Delta X_{R}^{(1)}\left(\omega_{\mathrm{ac}}\right)\right]^{2} \delta \mu_{R} .
\end{aligned}
$$

The first terms on the right-handside of Eqs. (36) and (37) refer to the case where there is no ac field.

Below, we present results for the sum of the two linearresponse coefficients,

$$
N^{(1)}\left(\omega_{\mathrm{ac}}\right)=N_{L}^{(1)}\left(\omega_{\mathrm{ac}}\right)+N_{R}^{(1)}\left(\omega_{\mathrm{ac}}\right)
$$

and

$$
\Delta X^{(1)}\left(\omega_{\mathrm{ac}}\right)=\left[\left[\Delta X_{L}^{(1)}\left(\omega_{\mathrm{ac}}\right)\right]^{2}+\left[\Delta X_{R}^{(1)}\left(\omega_{\mathrm{ac}}\right)\right]^{2}\right]^{1 / 2} .
$$

Both quantities are complex, since as mentioned the calculations use for the ac chemical potentials the form $\delta \mu_{L(R)} \exp \left[i \omega_{\mathrm{ac}} t\right]$; the phase delay of $N^{(1)}$ and $\Delta X^{(1)}$ yields the phase shift away from $\omega_{\text {ac }} t$. We plot below the absolute values of $N^{(1)}\left(\omega_{\mathrm{ac}}\right)$ and of $\Delta X^{(1)}\left(\omega_{\mathrm{ac}}\right)$, and the phase of the latter (see Ref. 38).

The absolute value of $N^{(1)}$ is plotted in Fig. 4(a) for a transparent junction whose transmission is 0.8, and in Fig. 5(a) for an opaque junction, with $\mathcal{T}=0.2$. Perhaps not surprisingly, the larger is the bias voltage $V$, the larger is $\left|N^{(1)}\right|$, for both types of junctions. This tendency was found also in the absence of the ac field ${ }^{57}$ Large values of $\omega_{\mathrm{ac}} / \omega_{0}$ suppress the effect of the ac field on the vibrations' occupation. Furthermore, for large $\Gamma,\left(\Gamma=2 \omega_{0}\right)$, the maximum value of $N$ is located at $\omega_{\mathrm{ac}}=0$. This value deceases monotonically with the ac frequency. For small $\Gamma,\left(\Gamma=0.5 \omega_{0}\right)$, the peak due to the resonance between the molecular vibration and the ac frequency emerges at $0<\omega_{\mathrm{ac}}<\omega_{0}$.

While the ac field-modified amplitude $\left|\Delta X^{(1)}\right|$, Fig. 4(b) for the high transmission and Fig. 5(b) for the lower one, also decays at high values of the ac frequency, this fluctuation possesses a distinct peak around $\omega_{\mathrm{ac}} \sim 2 \omega_{0}$, apparently of the same origin as the dip in the contribution of the twovibrational modes processes in the particle-hole propagator to $C\left(\omega_{\mathrm{ac}}\right)$, see Figs. 9(c) and 10(c). For small ac frequency, $\left|\Delta X^{(1)}\right|$ behaves similarly to $\left|N^{(1)}\right|$; the characteristics resemble those of the displacement $x$ of a classical forced oscillator when the decay rate is large. Indeed, the displacement $x$ of a classical forced-oscillator obeys the equation of motion

$$
\ddot{x}+\eta \dot{x}+\omega_{0}^{2} x=f \cos \left(\omega_{\mathrm{ac}} t\right),
$$

where $f$ is the force (normalized to the proper units). The amplitude $A$ of this driven operator is

$$
A=\frac{f}{\sqrt{\left(\omega_{0}^{2}-\omega_{\mathrm{ac}}^{2}\right)^{2}+\eta^{2} \omega_{\mathrm{ac}}^{2}}},
$$

and its phase shift $\theta$ is given by

$$
\tan \theta=\frac{\eta \omega_{\mathrm{ac}}}{\omega_{0}^{2}-\omega_{\mathrm{ac}}^{2}} .
$$
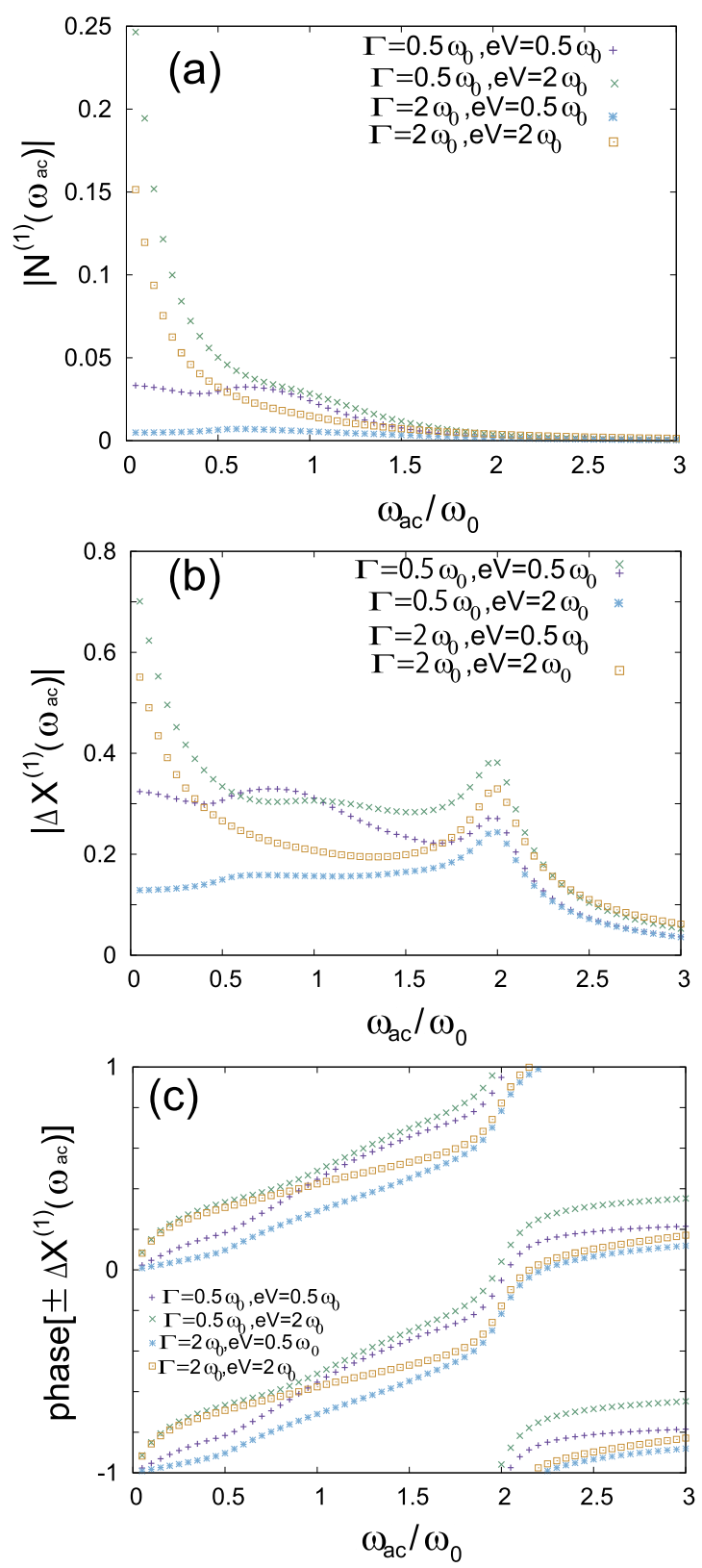

FIG. 4. A transparent junction, $\mathcal{T}=0.8$. (a) The change in the vibrations' population due to the ac field $\left[\left|N^{(1)}\right|\right.$, see Eq. (38)] scaled by $\omega_{0}^{-1}$, as a function of $\omega_{\mathrm{ac}} / \omega_{0}$; (b) the change in the displacement's fluctuation amplitude [in units of $\left(2 \omega_{0}\right)^{-1 / 2}$, see Eq. (39)], due to the ac field as a function of $\omega_{\mathrm{ac}} / \omega_{0}$; (c) the phase delay (in units of $\pi$ ) of the displacement's fluctuation.

Thus at $\omega_{\mathrm{ac}}=\omega_{0}$, the phase delay of the classical oscillator reaches $\pi / 2$ and "jumps" from being positive $\left(\omega_{0}^{2}>\omega_{\mathrm{ac}}^{2}\right)$ to a negative value. As seen in Figs. 4(c) and 5(c), the phase delay of the displacement is 0 or $-\pi$ at very small ac frequencies and reaches the out-of-phase configuration where it is $\pm \pi / 2$ around $\omega_{\mathrm{ac}} \approx \omega_{0}$. The fact that it occurs at higher frequencies, $\omega_{\mathrm{ac}}>\omega_{0}$ (e.g., for $\Gamma>\omega_{0}$ ), indicates that the resonance itself is shifted. In any event, one may understand this dependence on classical grounds. This is not the case for the additional structure at $\omega_{\mathrm{ac}} \sim 2 \omega_{0}$. As observed for the amplitude $\left|\Delta X^{(1)}\right|$, this feature is related to the two-vibrational mode processes contributing to the particle-hole propagator (see the Appendix); it cannot be explained by referring to the classical driven oscillator. This feature is thus a unique 

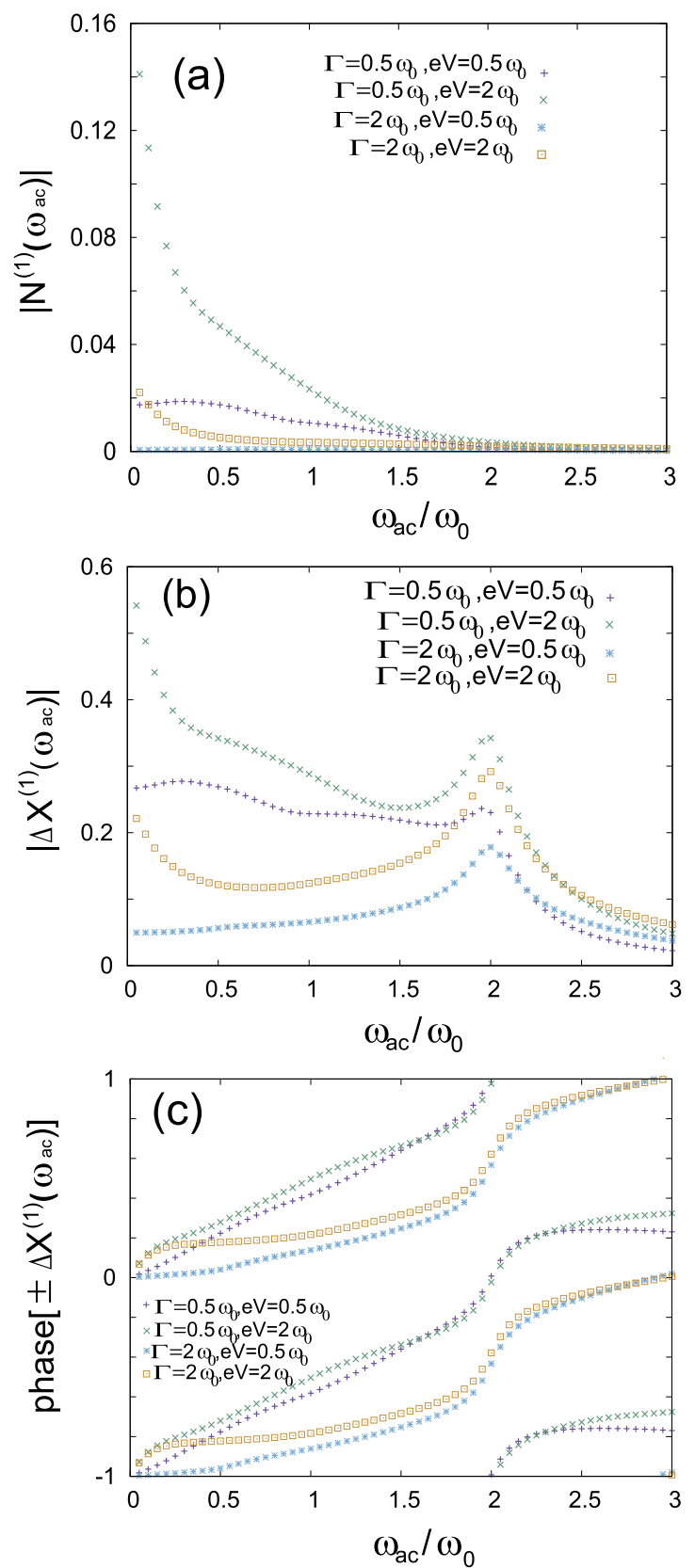

FIG. 5. An opaque junction, $\mathcal{T}=0.2$ (the other parameters are as in Fig. 4).

manifestation of an electro-mechanical interaction in the quantum regime.

\section{DC-CURRENT NOISE AND FULL-COUNTING STATISTICS}

The term full-counting statistics (FCS) refers to the distribution function of the probability $P_{\tau}(q)$ for the number $q$ of transmitted charges to traverse a quantum conductor during a certain time, $\tau$, at out-of-equilibrium conditions. We consider the FCS when no ac field is applied to the junction, $\delta \mu_{L}=\delta \mu_{R}=0$ in Eq. (1), and thus

$$
\mu_{L}-\mu_{R}=e V,
$$

where $V$ is the bias voltage (the frequency-dependent third cumulant is considered in Ref. 58). In our previous work, ${ }^{37}$ we have investigated the FCS for weak electron-vibration coupling, emphasizing in particular the fluctuation theorem (FT) and charge conservation. The FT has been recently applied to nonequilibrium quantum transport, ${ }^{59-68}$ including the present issue in the strong-coupling regime. ${ }^{45,47}$ The FT is a consequence of micro-reversibility and can be understood as a microscopic extension of the second law of thermodynamics. It relates the probabilities to find negative and positive entropy productions,

$$
P_{\tau}(-q)=P_{\tau}(q) e^{-\beta q e V} .
$$

Despite its simple appearance, Eq. (44) produces linearresponse results, i.e., it ensures the fluctuation-dissipation theorem and Onsager's reciprocal relations close to equilibrium, ${ }^{59-65}$ while conveying invaluable information at nonequilibrium conditions.

The Fourier transform of the probability distribution is connected to the Keldysh partition function, ${ }^{30}$

$$
\begin{aligned}
\sum_{q} P_{\tau}(q) e^{i q \lambda}= & \left\langle\tilde{T} \exp \left[i \int_{0}^{\tau} d t \mathcal{H}_{\mathrm{tun},-}(t)_{I}\right]\right. \\
& \left.\times T \exp \left[-i \int_{0}^{\tau} d t \mathcal{H}_{\mathrm{tun},+}(t)_{I}\right]\right\rangle \approx \exp [\tau \mathcal{F}(\lambda)],
\end{aligned}
$$

where $T(\tilde{T})$ is the (anti) time-ordering operator. The subscript $I$ indicates that time dependency is in the interaction picture [see Eq. (6)].

At steady state, that is, in the long measurement-time limit, $\tau \rightarrow \infty$, the cumulants of the current, e.g., the noise, are derived from the derivatives of the cumulant generating-function (CGF), $\mathcal{F}$,

$$
\left\langle\left\langle I^{n}\right\rangle\right\rangle=\left.e^{n} \frac{\partial^{n} \mathcal{F}(\lambda)}{\partial(i \lambda)^{n}}\right|_{\lambda=0} .
$$

As mentioned, our calculations are confined to the regime of a weak electron-vibration coupling energy and therefore require an expansion in $\gamma$. However, there is a subtle point in this procedure. A naïve second-order perturbation theory is not capable of producing the correct nonequilibrium distribution function of the vibrations. ${ }^{39,57}$ One has therefore to re-sum an infinite number of diagrams by adopting the linked cluster expansion (see, e.g., Ref. 56) or to use an even more advanced method, the nonequilibrium Luttinger-Ward functional, as exploited in Ref. 37. In terms of this functional, the CGF is

$$
\mathcal{F}(\lambda)=\mathcal{F}_{0}(\lambda)-\bar{\Phi}^{(2)}(\lambda)
$$

where $\mathcal{F}_{0}$ is the CGF of the noninteracting electrons (i.e., the CGF for $\gamma=0$ ),

$$
\mathcal{F}_{0}(\lambda)=\frac{1}{2 \pi \beta}\left[\left(\operatorname{arccosh} X_{\lambda}\right)^{2}\right]
$$

with

$$
X_{\lambda}=(1-\mathcal{T}) \cosh \frac{\beta e V}{2}+\mathcal{T} \cosh \frac{\beta e V+2 i \lambda}{2},
$$

where $\mathcal{T}$ is the transmission of the localized level. A trivial constant preserving the normalization condition, $\mathcal{F}_{0}(0)=0$, is omitted here for convenience. 
The effect of the electron-vibration interaction on the FCS is contained in the second term of Eq. (47). Using a diagrammatic expansion in powers of the small parameter

$$
g=2 \gamma^{2} /\left(\pi \Gamma^{2}\right),
$$

where $\Gamma$, Eq. (19), is the width of the resonance, and Ref. 37 shows that $\bar{\Phi}^{(2)}$ is faithfully described within the randomphase approximation (RPA),

$$
\bar{\Phi}^{\mathrm{RPA}}(\lambda)=\bar{\Phi}^{(2)}(\lambda)+\mathcal{O}\left(g^{2}\right)
$$

up to second order in $\gamma$, i.e., up to first order in $g$, Eq. (50). In terms of the vibration Green's function $D_{\lambda}(\omega)$,

$$
\bar{\Phi}^{\mathrm{RPA}}(\lambda)=\frac{1}{4 \pi} \int d \omega \ln \operatorname{det} D_{\lambda}^{-1}(\omega) .
$$

Here,

$$
D_{\lambda}^{-1}(\omega)=\left[\begin{array}{cc}
\frac{\omega^{2}-\omega_{0}^{2}}{2 \omega_{0}}-\Pi_{\lambda}^{++}(\omega) & \Pi_{\lambda}^{+-}(\omega) \\
\Pi_{\lambda}^{-+}(\omega) & \frac{\omega_{0}^{2}-\omega^{2}}{2 \omega_{0}}-\Pi_{\lambda}^{--}(\omega)
\end{array}\right],
$$

where $\Pi$ is the electron-hole propagator, resulting from the polarization of the electrons [Eqs. (29) and (30), modified in a non-trivial way to include the effect of the counting field $\lambda$, see Ref. 37 for details].

Figure 6(a) displays the average current at a finite temperature (solid curves) and at zero temperature (dashed curves), as a function of the bias voltage, for a spatially symmetric junction. (Temperature is measured in units of $\omega_{0}$ and the bias is scaled by $\omega_{0} / e$.) When the transmission is perfect, $\mathcal{T}=1$, the current is suppressed once $|e V|>\omega_{0}$, since the electrons can then be backscattered inelastically by the vibrations.

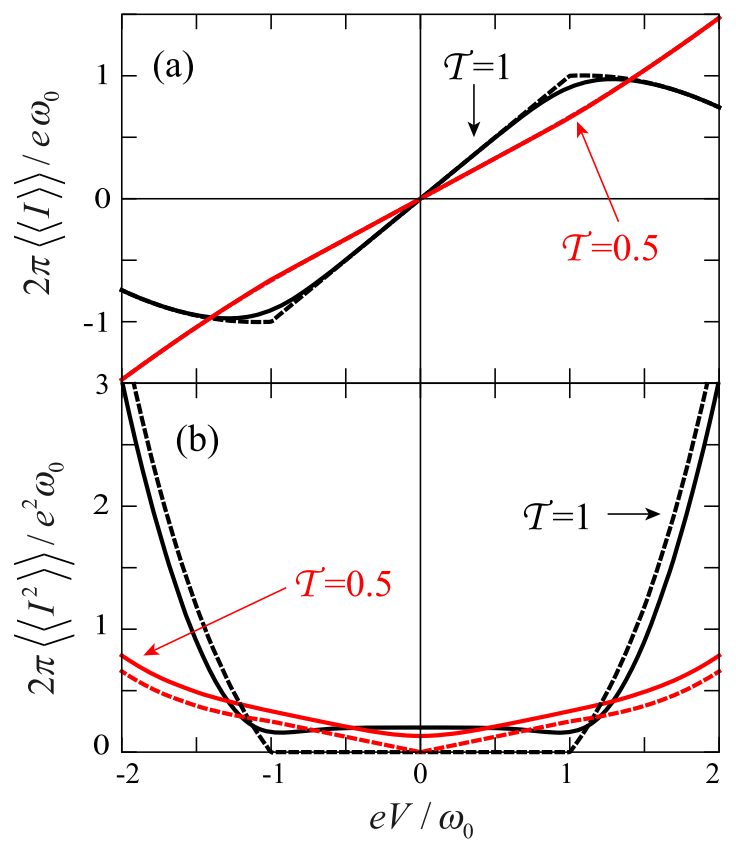

FIG. 6. The source-drain bias voltage dependence of the current (a) and of the current noise (b), for a perfect transmission, $\mathcal{T}=1$ (black curves), and for $\mathcal{T}=0.5$ (red curves). The solid curves are for $\beta \omega_{0}=10$, and the dashed ones are for zero temperature. The electron-vibration coupling constant $g$ [Eq. (50)] is 0.1. Adapted with permission from Utsumi et al., Phys. Rev. B 87, 115407 (2013). Copyright 2013 The American Physical Society.
At weaker transmissions, e.g., $\mathcal{T}=0.5$, the current is slightly enhanced above this threshold..$^{39}$ Apparently in this regime the main effect of the inelastic scattering of the electrons by the vibrations is to add more transport channels and to increase the phase-space volume available for the scattering events. A finite temperature tends to smear the kink structure of the $\mathcal{T}=1$ curve. The effect of the temperature on the current for $\mathcal{T}=0.5$ is rather insignificant.

Figure 6(b) depicts current noise, $\left\langle\left\langle I^{2}\right\rangle\right\rangle$, Eq. (46) with $n=2$. At perfect transmission and zero temperature, the noise vanishes below the threshold, at $|e V|<\omega_{0}$. Thermal fluctuations which arise at a finite temperature induce additional noise there. Although the current, i.e., $\langle\langle I\rangle\rangle$, is suppressed above the threshold, the inelastic scattering resulting from the interaction of the charge carriers with the vibrations enhances the noise in that regime. As in Fig. 6(a), the effect of the temperature on the noise in an imperfect junction $(\mathcal{T}=0.5)$ is far less dramatic, and the noise is simply enhanced.

As mentioned, the CGF is constructed from the long-time limit of the probability distribution $P_{\tau}(q)$ of a charge $e q$ passing through the junction during a time $\tau$. The approach of this probability to its steady-state value is characterized by the rate function, defined as $-\lim _{\tau \rightarrow \infty} \ln P_{\tau}(\tau I) / \tau$, where in the long-time limit the charge $e q$ is scaled as $I \tau$. Figure 7 displays the CGF and the rate function at perfect transmission. For comparison, we plot the corresponding curves for
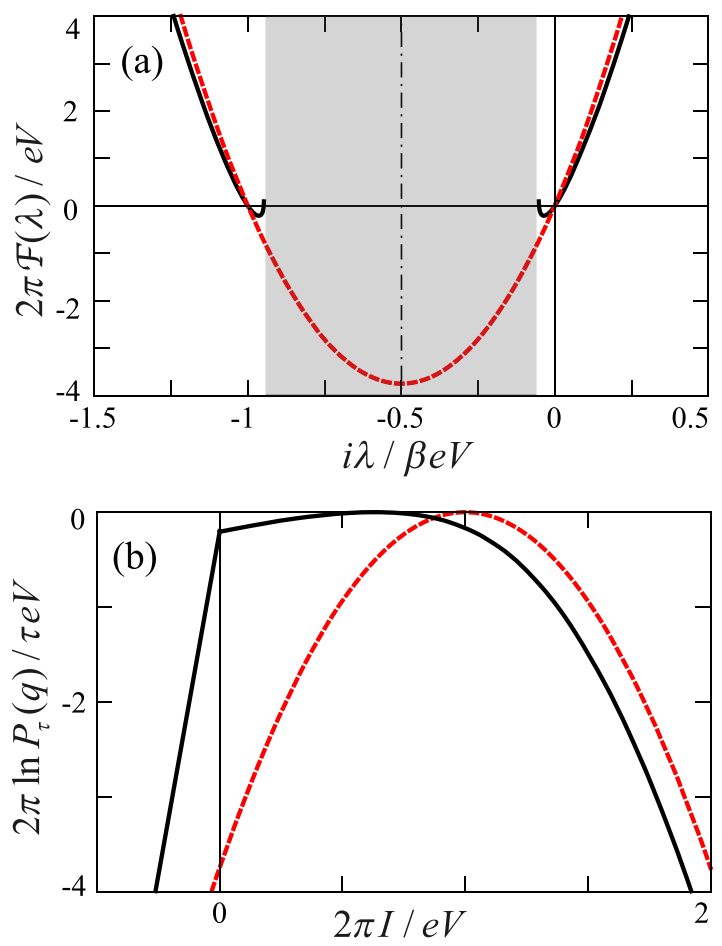

FIG. 7. The CGF [as a function of $i \lambda /(\beta e V)]$ (a), and the rate function [as a function of $I \propto q / \tau /(e V /(2 \pi))$ ] (b), for a perfect transmission, $\mathcal{T}=1$, $e V / \omega_{0}=1.5$, and $\beta \omega_{0}=10$. The solid (black) and the dotted (red) curves show results with $(g=0.1)$ and without $(g=0)$ the electron-vibration coupling, respectively. In the dashed area of panel (a), the CGF of interacting electrons is non-analytic and non-convex, resulting in a non-differentiable point of the rate function at $I=0$ (b). Adapted with permission from Utsumi et al., Phys. Rev. B 87, 115407 (2013). Copyright 2013 The American Physical Society. 
noninteracting electrons. ${ }^{37}$ The width of the rate function is enhanced by inelastic phonon scattering [Fig. 7(b)]. The CGF obeys the fluctuation theorem: the curves are symmetric around the dotted-dashed vertical line at $i \lambda=-\beta e V / 2$ [Fig. 7(a)]. The peak of the probability distribution is shifted in the negative direction and the probability to find large current fluctuations is suppressed as compared with that pertaining to noninteracting electrons [Fig. 7(b)]. In the shaded area of Fig. 7(a), the CGF is non-analytic and non-convex. Correspondingly, the rate function has a non-differentiable point at $I=0$ [see Fig. 7(b)]. As a result, although the probability to observe currents smaller than the average value is enhanced by the inelastic scattering due to the coupling with the vibrations, the probability to find negative currents $I<0$ is strongly reduced. This is consistent with the fluctuation theorem, which states that although thermal agitations generate current flowing in the opposite direction to the source-drain bias, that probability is exponentially suppressed at low temperatures.

The analogy between the characteristic function Eq. (45) and the equilibrium partition function helps to utilize statistical-physics methods for characterizing statistical properties of current fluctuations. The large-deviation approach connects the non-convexity in Fig. 7(a) and the kink in Fig. 7(b) with those observed in the Helmholtz free energy and the Gibbs free energy at the liquid-gas first-order phase transition. For noninteracting particles, zeros of the characteristic function (45) reside on the negative real axis in the complex $z=\exp [i \lambda]$ plane, ${ }^{69}$ reminiscent of the Yang-Lee zeros. ${ }^{70}$ The zeros' distribution can be used to characterize the interaction. ${ }^{71}$ In Ref. 37 it is found that the distribution of the singularities of $\mathcal{F}$ in the complex $\lambda$-plane can be a useful tool for identifying the effect of the electron-vibration interaction on the current probability distribution.

\section{CONCLUSIONS}

Our work is centered on nonequilibrium features in the dynamics of molecular junctions, in the regime of weak electron-vibration coupling. Specifically, we have presented a detailed study on the response of the junction to a weak ac field, in the presence of an arbitrary bias voltage (constant in time). In Sec. II we have analyzed the electronic response of the charge carriers to an ac field, and the modifications introduced by that field in the vibrations' distribution and in the fluctuations of the displacements, as a function of the ac frequency $\omega_{\mathrm{ac}}$. Perhaps the most intriguing feature we find is the newly discovered (relatively) sharp structure around $\omega_{\mathrm{ac}} \sim 2 \omega_{0}\left(\omega_{0}\right.$ is the frequency of the vibrational mode). While the structure around $\omega_{\mathrm{ac}} \sim \omega_{0}$ can be understood on a classical ground, the one at $\omega_{\mathrm{ac}} \sim 2 \omega_{0}$ is related to quantum effects in the particle-hole propagator (see Secs. II C and II D, and the Appendix). It is thus a unique manifestation of an electro-mechanical feature in coherent transport through a single-channel molecular junction. The effect of the arbitrary bias voltage is dwelled upon in particular in Secs. II B and III. Here we identify a change in the behavior of the dc current and its noise when the voltage is around $\omega_{0}$ (using units in which $\hbar=1$ and $e=1$ ). We hope that a future study on the fullcounting statistics and the cumulant generating-function when the junction is also placed in an ac field will substantiate our understanding of the dynamics of the excitations in molecular junctions.

\section{ACKNOWLEDGMENTS}

We thank Hiroshi Imamura for useful discussions. This work was supported by JSPS KAKENHI Grant Nos. JP26220711 and 6400390, by the Israel Science Foundation (ISF), and by the infrastructure program of Israel Ministry of Science and Technology under Contract No. 3-11173.

\section{APPENDIX: THE AC LINEAR-RESPONSE COEFFICIENT}

The diagrams that give the coefficient $C\left(\omega_{\mathrm{ac}}\right)$ [Eq. (17)] are depicted in Fig. 8. Figure 8(a) illustrates the diagrams that contribute to $C\left(\omega_{\mathrm{ac}}\right)$ : (i) is the contribution when the electron-vibration interaction is omitted, (ii) is that of the exchange term, and (iii) and (iv) pertain to the vertex corrections (due to the exchange term and to the particle-hole propagator, respectively).

In the presence of the ac field, the vibration Green's functions take the forms

$$
D^{r / a}\left(\omega_{\mathrm{ac}}, \omega\right)=D^{r / a}(\omega)+D^{(\mathrm{ac}) r / a}\left(\omega_{\mathrm{ac}}, \omega\right)
$$

and

$$
D^{<}\left(\omega_{\mathrm{ac}}, \omega\right)=D^{<}(\omega)+D^{(\mathrm{ac})<}\left(\omega_{\mathrm{ac}}, \omega\right)
$$

(a)
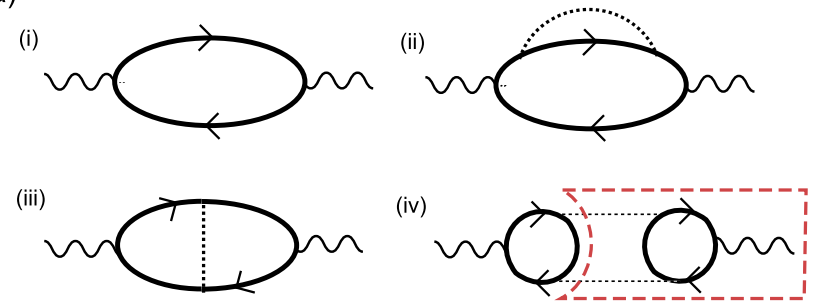

(b)

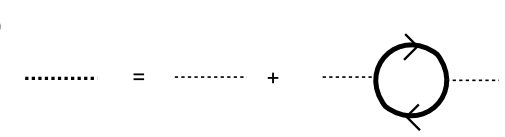

(c)

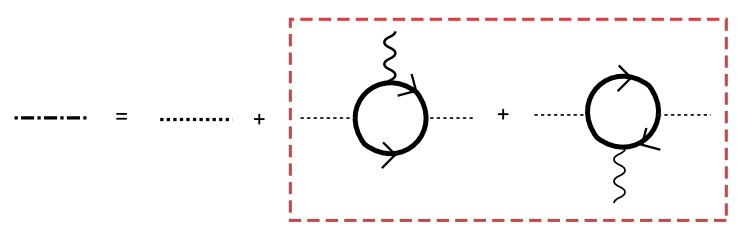

FIG. 8. (a) The diagrams for the ac transport coefficient $C\left(\omega_{\mathrm{ac}}\right)$, Eq. (17). The solid lines pertain to the electron Green's function and the dotted lines are the vibration Green's function; the wavy curve represents the ac external field. (i) Without the electron-vibration coupling; (ii) the exchange term (without the effect of the ac field in the vibration Green's function); (iii) vertex corrections for the exchange term (without the effect of the ac field in the vibration Green's function); (iv) particle-hole propagator (with the effect of the ac field in the vibration Green's function, enclosed in the dotted (red) lines). ((b) and (c)) The vibration Green's function; (b) in the absence of the ac field and (c) with the effect of the ac field. The latter, enclosed by the dashed (red) lines, appears due to the presence of the ac field and affects diagram (iv) in part (a). 
The first terms on the right-handside of Eqs. (A1) and (A2) are depicted in Fig. 8(b),

$D^{r / a}(\omega)=D^{(0) r / a}(\omega)+D^{(0) r / a}(\omega) \Pi^{r / a}(\omega) D^{(0) r / a}(\omega)$

and

$$
\begin{aligned}
D^{<}(\omega)= & D^{(0)<}(\omega)+D^{(0) r}(\omega) \Pi^{r}(\omega) D^{(0)<}(\omega) \\
& +D^{(0) r}(\omega) \Pi^{<}(\omega) D^{(0) a}(\omega) \\
& +D^{(0)<}(\omega) \Pi^{a}(\omega) D^{(0) a}(\omega) .
\end{aligned}
$$

The second terms on the right-handside of Eqs. (A1) and (A2), which are due to the ac field, are shown in Fig. 8(c),

$$
D^{(\mathrm{ac}) r / a}\left(\omega_{\mathrm{ac}}, \omega\right)=D^{(0) r / a}\left(\omega-\omega_{\mathrm{ac}}\right) \Pi^{(\mathrm{ac}) r / a}\left(\omega_{\mathrm{ac}}, \omega\right) D^{(0) r / a}(\omega)
$$

and

$$
\begin{aligned}
D^{(\mathrm{ac})<}\left(\omega_{\mathrm{ac}}, \omega\right)= & D^{(0) r}\left(\omega-\omega_{\mathrm{ac}}\right) \Pi^{(\mathrm{ac}) r}\left(\omega_{\mathrm{ac}}, \omega\right) D^{(0)<}(\omega) \\
& +D^{(0) r}\left(\omega-\omega_{\mathrm{ac}}\right) \Pi^{(\mathrm{ac})<}\left(\omega_{\mathrm{ac}}, \omega\right) D^{(0) a}(\omega) \\
& +D^{(0)<}\left(\omega-\omega_{\mathrm{ac}}\right) \Pi^{(\mathrm{ac}) a}\left(\omega_{\mathrm{ac}}, \omega\right) D^{(0) a}(\omega) .
\end{aligned}
$$

Here, $D^{(0)}$ is the bare vibrational modes' Green's function,

$$
D^{(0) r / a}(\omega)=\frac{1}{\omega-\omega_{0} \pm i \eta / 2}-\frac{1}{\omega+\omega_{0} \pm i \eta / 2}
$$

and

$$
\begin{aligned}
D^{(0)<}(\omega)= & -i\left(\frac{\eta}{\left(\omega+\omega_{0}\right)^{2}+(\eta / 2)^{2}}(N+1)\right. \\
& \left.+\frac{\eta}{\left(\omega-\omega_{0}\right)^{2}+(\eta / 2)^{2}} N\right),
\end{aligned}
$$

where $N$ denotes the vibrations' population. Since Eq. (A8) is zeroth-order in the electron-vibration coupling, then in principle, $N$ at a finite temperature is determined by the coupling to a surrounding phonon bath ${ }^{57}$ Here we confine ourselves to zero temperature, and hence the zeroth-order vibration Green's function contains $N=0$. The processes in which a vibrational mode excites a particle-hole pair and then becomes the vibrational mode again are given in Eqs. (A1) and (A2). The electron-hole propagator determines the lifetime of the vibrational mode, when the system is effectively decoupled from an external phonon bath [see the discussion in the paragraph following Eq. (30)]. The expressions for the particle-hole propagator in the absence of the ac field are given in Eqs. (29) and (30), and the ones in the presence of the ac field are

$$
\Pi^{(\mathrm{ac}) r / a}\left(\omega_{\mathrm{ac}}, \omega\right)=-i \gamma^{2} \int \frac{d \omega^{\prime}}{2 \pi}\left(G_{00}^{(\mathrm{ac})(0)<}\left(\omega_{\mathrm{ac}}, \omega^{\prime}\right) G_{00}^{(0) a}\left(\omega^{\prime}-\omega\right)+G_{00}^{(0) r}\left(\omega^{\prime}-\omega_{\mathrm{ac}}\right) G_{00}^{(\mathrm{ac})(0)<}\left(\omega_{\mathrm{ac}}, \omega^{\prime}-\omega\right)\right)
$$

and

$$
\Pi^{(\mathrm{ac})<}\left(\omega_{\mathrm{ac}}, \omega\right)=-i \gamma^{2} \int \frac{d \omega^{\prime}}{2 \pi}\left(G_{00}^{(\mathrm{ac})(0)<}\left(\omega_{\mathrm{ac}}, \omega^{\prime}\right) G_{00}^{(0)>}\left(\omega^{\prime}-\omega\right)+G_{00}^{(0)<}\left(\omega^{\prime}-\omega_{\mathrm{ac}}\right) G_{00}^{(\mathrm{ac})(0)<}\left(\omega_{\mathrm{ac}}, \omega^{\prime}-\omega\right)\right),
$$

where $G_{00}^{(\text {ac)(0)< }}$ is given in Eq. (32) (for a symmetric junction).

For concreteness, we give the detailed expressions of the diagrams for a spatially symmetric junction, i.e., $\Gamma_{L}=\Gamma_{R}=\Gamma / 2$ in Eq. (19). Diagram (i) in Fig. 8(a) pertains to the case where the electron-vibration coupling is absent and reads

$$
C^{(0)}\left(\omega_{\mathrm{ac}}\right)=\frac{e}{4 \omega_{\mathrm{ac}}} \operatorname{Re}\left[\int \frac{d \omega}{2 \pi} i \Gamma\left[f_{L}\left(\omega-\omega_{\mathrm{ac}}\right)+f_{R}\left(\omega-\omega_{\mathrm{ac}}\right)-f_{L}(\omega)-f_{R}(\omega)\right]\left[G_{00}^{(0) r}\left(\omega-\omega_{\mathrm{ac}}\right)-G_{00}^{(0) a}(\omega)\right]\right],
$$

where the electron Green's function $G_{00}^{(0)}$ is given by Eq. (24). The exchange contribution, diagram (ii) in Fig. 8(a), reads

$$
C^{(\mathrm{ex})}\left(\omega_{\mathrm{ac}}\right)=\frac{e}{4 \omega_{\mathrm{ac}}} \operatorname{Re}\left[\int \frac{d \omega}{2 \pi} i \Gamma\left[f_{L}\left(\omega-\omega_{\mathrm{ac}}\right)+f_{R}\left(\omega-\omega_{\mathrm{ac}}\right)-f_{L}(\omega)-f_{R}(\omega)\right]\left[G_{00}^{(\mathrm{ex}) r}\left(\omega-\omega_{\mathrm{ac}}\right)-G_{00}^{(\mathrm{ex}) a}(\omega)\right]\right],
$$

where

$$
G_{00}^{(\mathrm{ex}) r / a}(\omega)=i \gamma^{2}\left[G_{00}^{(0) r / a}(\omega)\right]^{2} \int \frac{d \omega^{\prime}}{2 \pi}\left[G_{00}^{(0)<}\left(\omega-\omega^{\prime}\right) D^{r / a}\left(\omega^{\prime}\right)+G_{00}^{(0) r / a}\left(\omega-\omega^{\prime}\right) D^{<}\left(\omega^{\prime}\right) \pm G_{00}^{(0) r / a}\left(\omega-\omega^{\prime}\right) D^{r / a}\left(\omega^{\prime}\right)\right]
$$

[see Eqs. (A1)-(A6)]. The expressions for the third diagram [denoted (iii) in Fig. 8(a)] and the fourth one [denoted (iv) in Fig. $8(a)]$ represent vertex corrections to the transport coefficient. They both are given by

$$
\begin{aligned}
C_{\ell}^{(\mathrm{ver})}\left(\omega_{\mathrm{ac}}\right)= & -\frac{e}{4 \delta \mu} \operatorname{Re}\left[\int \frac { d \omega } { 2 \pi } i \Gamma \left(G_{00}^{(0) r}\left(\omega-\omega_{\mathrm{ac}}\right) \Sigma_{\ell}^{(\mathrm{ver}) r}\left(\omega_{\mathrm{ac}}, \omega\right) G_{00}^{(0) r}(\omega)\left[f_{L}(\omega)-f_{R}(\omega)\right]\right.\right. \\
& \left.\left.-G_{00}^{(0) a}\left(\omega-\omega_{\mathrm{ac}}\right) \Sigma_{\ell}^{(\mathrm{ver}) a}\left(\omega_{\mathrm{ac}}, \omega\right) G_{00}^{(0) a}(\omega)\left[f_{L}\left(\omega-\omega_{\mathrm{ac}}\right)-f_{R}\left(\omega-\omega_{\mathrm{ac}}\right)\right]\right)\right] .
\end{aligned}
$$

For the diagram (iii) in Fig. 8(a) $\Sigma_{\ell}^{(\mathrm{ver}) r / a}\left(\omega_{\mathrm{ac}}, \omega\right)=\Sigma_{1}^{(\mathrm{ver}) r / a}\left(\omega_{\mathrm{ac}}, \omega\right)$, where

$$
\Sigma_{1}^{(\mathrm{ver}) r / a}\left(\omega_{\mathrm{ac}}, \omega\right)=i \gamma^{2} \int \frac{d \omega^{\prime}}{2 \pi} G_{00}^{(\mathrm{ac})(0)<}\left(\omega_{\mathrm{ac}}, \omega-\omega^{\prime}\right) D^{r / a}\left(\omega^{\prime}\right),
$$


and consequently the contribution of that diagram is denoted $C_{1}^{(\mathrm{ver})}$. [The electron Green's functions appearing in Eq. (A15) are given in Eq. (32).] Diagram (iv) in Fig. 8(a) is given by Eq. (A14), with $\Sigma_{\ell}^{(\mathrm{ver}) r / a}\left(\omega_{\mathrm{ac}}, \omega\right)=\Sigma_{2}^{(\mathrm{ver}) r / a}\left(\omega_{\mathrm{ac}}, \omega\right)$, and is denoted $C_{2}^{(\mathrm{ver})}$, where

$\Sigma_{2}^{(\mathrm{ver}) r / a}\left(\omega_{\mathrm{ac}}, \omega\right)=i \gamma^{2} \int \frac{d \omega^{\prime}}{2 \pi}\left(\left[G_{00}^{(0)<}\left(\omega-\omega^{\prime}\right) \pm G_{00}^{(0) r / a}\left(\omega-\omega^{\prime}\right)\right] D^{(\mathrm{ac}) r / a}\left(\omega_{\mathrm{ac}}, \omega^{\prime}\right)+G_{00}^{(0) r / a}\left(\omega-\omega^{\prime}\right) D^{(\mathrm{ac})<}\left(\omega_{\mathrm{ac}}, \omega^{\prime}\right)\right)$.

In the numerical calculations, the rate $\eta$ [see Eq. (27) and the discussion following Eq. (30)] was chosen to be $0.1 \omega_{0}$; as a result, it became larger than $\operatorname{Im} \Pi^{a}(\omega)$ and therefore the vibration Green's functions in Eqs. (A13) and (A15) could be replaced by $D^{(0)}$.

It is illuminating to study the effect of the electronvibration interaction on each diagram separately. Figures 9(a) and 10(a) display the contribution of the exchange diagram,
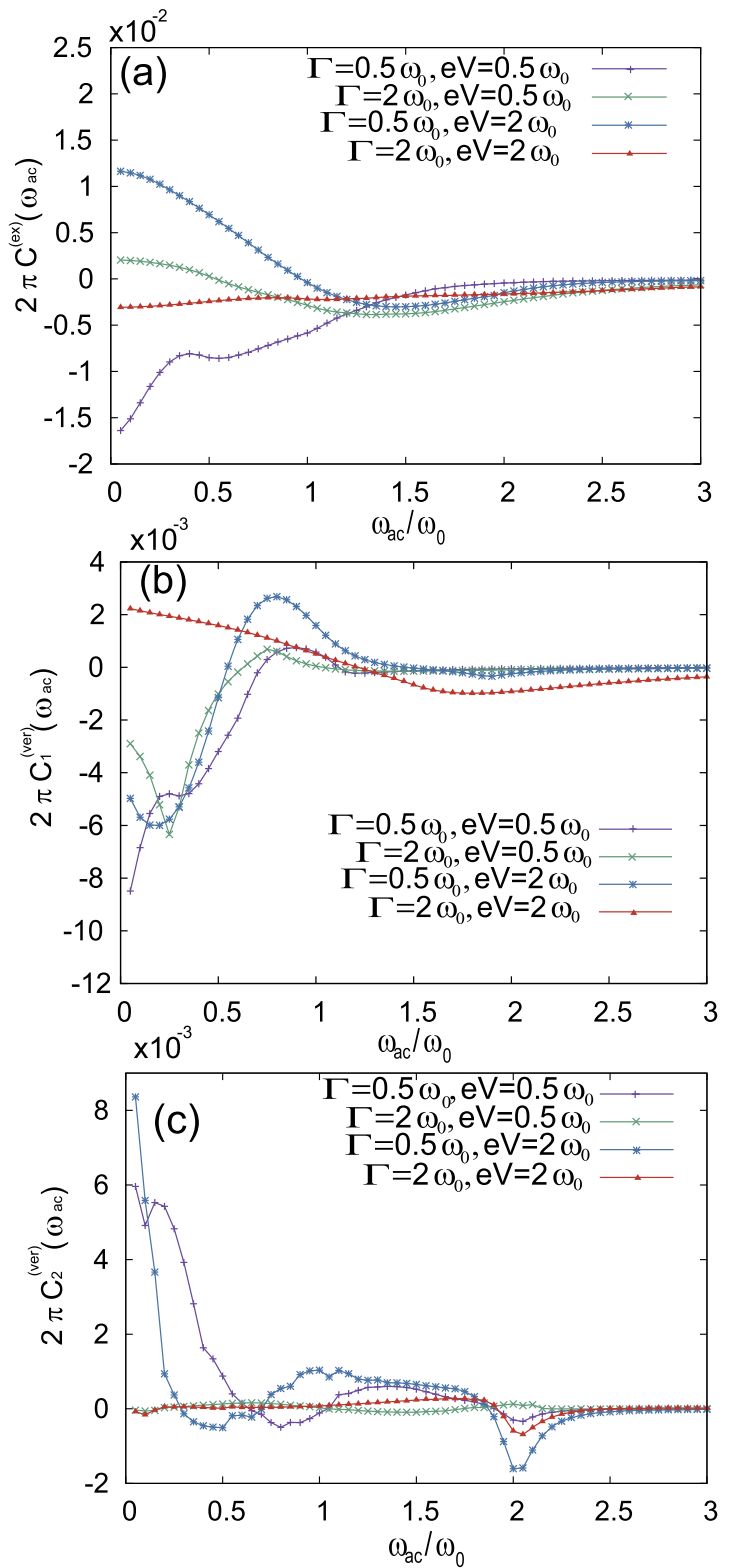

FIG. 9. The separate contributions of the various diagrams to $C\left(\omega_{\mathrm{ac}}\right)$, as functions of $\omega_{\mathrm{ac}} / \omega_{0}$, for a transparent junction, $\mathcal{T}=0.8$. (a) $C^{(\mathrm{ex})}$, Eq. (A12); (b) diagram (iii), Eq. (A14) for $i=1$; (c) diagram (iv), Eq. (A14) for $i=2$. (ii) in Fig. 8(a). This diagram represents the dressing of the electron Green's function by the emission and absorption of vibrational modes. At zero temperature, and when the bias voltage is smaller than $\omega_{0}$, the electrons can only absorb and emit virtual vibrational modes; this gives rise to a shift in the energy of the localized level. [This shift differs from the one caused by the Hartree self-energy, Eq. (22), which is ignored here.] The change in the localized-level energy can increase or decrease the contribution of this diagram at small ac frequencies (as compared to $\left.\omega_{0}\right)$. By comparing Fig. 9(a) (for $\mathcal{T}=0.8$ )
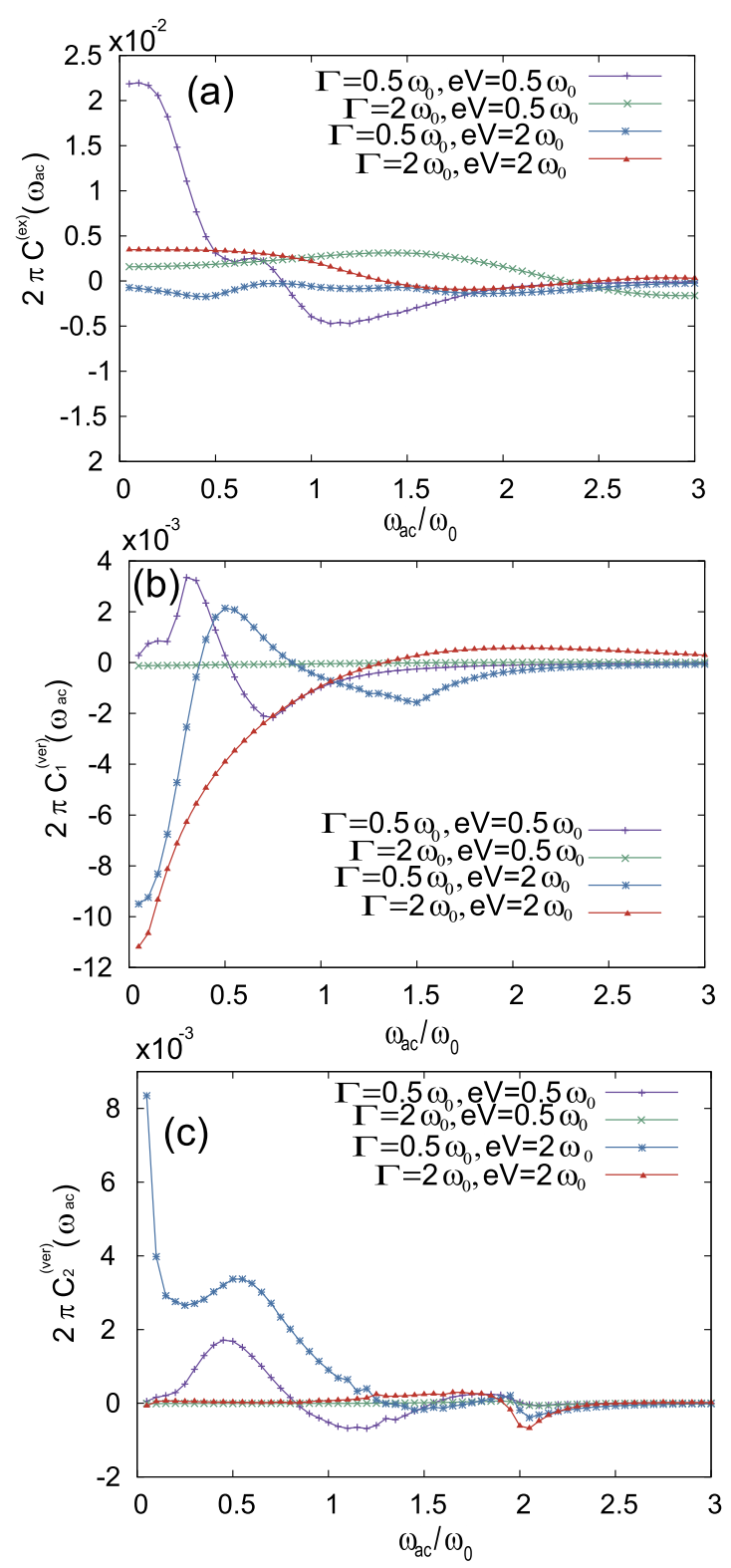

FIG. 10. Same as Fig. 9 with $\mathcal{T}=0.2$. 
with Fig. 10(a) (for $\mathcal{T}=0.2$ ), it is seen that at $e V=0.5 \omega_{0}$ and with a small resonance width, $\Gamma=0.5 \omega_{0}, C^{(\mathrm{ex})}$ is negative for $\mathcal{T}=0.8$ and is positive for $\mathcal{T}=0.2$. The opposite, but rather weaker, tendency is found for a wide resonance, $\Gamma=2 \omega_{0}$. At larger biases, e.g., $\mathrm{eV}=2 \omega_{0}$, real inelastic electron-vibration scattering processes are possible. These broaden the line width of the localized level. However, modifying the resonance width and/or the transmission does not lead to significant effects. This is also the situation at higher values of $\omega_{\mathrm{ac}}$.

The contribution of diagram (iii) in Fig. 8(a) is shown in Figs. 9(b) and 10(b). This diagram corresponds to the processes in which an electron and a hole exchange vibrational modes. This process results in a negative contribution of diagram (iii) at small values of $\omega_{\text {ac }}$, except when $\Gamma=e V=2 \omega_{0}$ and the junction is transparent, Fig. 9(b), and when $\Gamma=e V=0.5 \omega_{0}$ and the junction is opaque, Fig. 10(b). For both types of junctions, there appears a peak in the contribution of this diagram for the larger bias voltages, $e V=2 \omega_{0}$, just below $\omega_{\mathrm{ac}}=\omega_{0}$; it may reflect a resonance in the particle-hole channel around this frequency.

The behavior of the contribution of diagram (iv) of Fig. 8(a) is depicted in Figs. 9(c) and 10(c). This diagram describes two-vibration exchange between the electrons [see Fig. 8(a)]. There are two distinct features in the contribution of this diagram. First, its contribution at small ac frequencies is positive, provided that the resonance width $\Gamma$ is less than $\omega_{0}$ (otherwise, it is negligibly small). Second, it shows a dip at $\omega_{\mathrm{ac}} \sim 2 \omega_{0}$. Apparently, the appearance of this dip, which disappears at zero bias, ${ }^{38}$ can be attributed to two-phonon processes that reach resonance conditions at this frequency.

${ }^{1}$ C. Joachim, J. K. Gimzewski, and A. Aviram, "Electronics using hybrid-molecular and mono-molecular devices," Nature 408, 541 (2000); J. C. Cuevas and E. Scheer, Molecular Electronics (World Scientific, Singapore, 2010).

${ }^{2}$ H. Park, J. Park, A. K. L. Lim, E. H. Anderson, A. P. Alivisatos, and P. L. McEuen, "Nanomechanical oscillations in a single- $C_{60}$ transistor," Nature 407, 57 (2000); V. Sazonova, Y. Yaish, H. Üstünel, D. Roundy, T. A. Arias, and P. L. McEuen, "A tunable carbon nanotube electromechanical oscillator," ibid. 431, 284 (2004).

${ }^{3}$ R. H. M. Smit, Y. Noat, C. Untiedt, N. D. Lang, M. C. van Hemert, and J. M. van Ruitenbeek, "Measurement of the conductance of a hydrogen molecule," Nature 419, 906 (2002).

${ }^{4}$ M. Kiguchi, O. Tal, S. Wohlthat, F. Pauly, M. Krieger, D. Djukic, J. C. Cuevas, and J. M. van Ruitenbeek, "Highly conductive molecular junctions based on direct binding of benzene to platinum electrodes," Phys. Rev. Lett. 101, 046801 (2008).

${ }^{5}$ S. Sapmaz, P. Jarillo-Herrero, Ya. M. Blanter, C. Dekker, and H. S. J. van der Zant, "Tunneling in suspended carbon nanotubes assisted by longitudinal phonons,” Phys. Rev. Lett. 96, 026801 (2006); R. Leturcq, C. Stampfer, K. Inderbitzin, L. Durrer, C. Hierold, E. Mariani, M. G. Schultz, F. von Oppen, and K. Ensslin, "Franck-Condon blockade in suspended carbon nanotube quantum dots," Nat. Phys. 5, 327 (2009).

${ }^{6}$ O. Tal, M. Krieger, B. Leerink, and J. M. van Ruitenbeek, "Electronvibration interaction in single-molecule junctions: From contact to tunneling regimes," Phys. Rev. Lett. 100, 196804 (2008).

${ }^{7}$ N. Agraït, C. Untiedt, G. Rubio-Bollinger, and S. Vieira, "Onset of energy dissipation in ballistic atomic wires," Phys. Rev. Lett. 88, 216803 (2002); M. Kumar, R. Avriller, A. Levy Yeyati, and J. M. van Ruitenbeek, "Detection of vibration-mode scattering in electronic shot noise," ibid. 108, 146602 (2012).

${ }^{8}$ S. Ballmann, R. Härtle, P. B. Coto, M. Elbing, M. Mayor, M. R. Bryce, M. Thoss, and H. B. Weber, "Experimental evidence for quantum interference and vibrationally induced decoherence in single-molecule junctions," Phys. Rev. Lett. 109, 056801 (2012).
${ }^{9}$ S. V. Aradhya and L. Venkataraman, "Single-molecule junctions beyond electronic transport," Nat. Nano. 8, 399 (2013).

${ }^{10}$ Y. Kim, H. Song, F. Strigl, H. Pernau, T. Lee, and E. Scheer, "Conductance and vibrational states of single-molecule junctions controlled by mechanical stretching and material variation," Phys. Rev. Lett. 106, 196804 (2011).

${ }^{11}$ D. R. Ward, N. J. Halas, J. W. Ciszek, J. M. Tour, Y. Wu, P. Nordlander, and D. Natelson, "Simultaneous measurements of electronic conduction and Raman response in molecular junctions," Nano Lett. 8, 919 (2008).

${ }^{12}$ J. Koch, M. Semmelhack, F. von Oppen, and A. Nitzan, "Current-induced nonequilibrium vibrations in single-molecule devices," Phys. Rev. B 73, 155306 (2006).

${ }^{13}$ K. Kaasbjerg, T. Novotný, and A. Nitzan, "Charge-carrier-induced frequency renormalization, damping, and heating of vibrational modes in nanoscale junctions," Phys. Rev. B 88, 201405(R) (2013).

${ }^{14}$ D. R. Ward, D. A. Corley, J. M. Tour, and D. Natelson, "Vibrational and electronic heating in nanoscale junctions," Nat. Nano. 6, 33 (2011).

${ }^{15}$ L. Rincón-Garca, C. Evangeli, G. Rubio-Bollinger, and N. Agraït, "Thermopower measurements in molecular junctions," Chem. Soc. Rev. 45, 4285 (2016).

${ }^{16}$ Y. Li, P. Zolotavin, P. Doak, L. Kronik, J. B. Neaton, and D. Natelson, "Interplay of bias-driven charging and the vibrational Stark effect in molecular junctions," Nano Lett. 16, 1104 (2016).

${ }^{17}$ D. Rakhmilevitch, R. Korytár, A. Bagrets, F. Evers, and O. Tal, "Electronvibration interaction in the presence of a switchable Kondo resonance realized in a molecular junction," Phys. Rev. Lett. 113, 236603 (2014).

${ }^{18}$ A. Mugarza, C. Krull, R. Robles, S. Stepanow, G. Ceballos, and P. Gambardella, "Spin coupling and relaxation inside molecule-metal contacts," Nat. Commun. 2, 490 (2011).

${ }^{19}$ M. Paulsson, T. Frederiksen, and M. Brandbyge, "Inelastic transport through molecules: Comparing first-principles calculations to experiments," Nano Lett. 6, 258 (2006).

${ }^{20}$ W. R. French, C. R. Iacovella, I. Rungger, A. M. Souza, S. Sanvito, and P. T. Cummings, "Atomistic simulations of highly conductive molecular transport junctions under realistic conditions," Nanoscale 5, 3654 (2013).

${ }^{21}$ Z.-F. Liu and J. B. Beaton, "Communication: Energy-dependent resonance broadening in symmetric and asymmetric molecular junctions from an ab initio non-equilibrium Green's function approach,” J. Chem. Phys. 141, 131104 (2014).

${ }^{22}$ M. Bai, C. S. Cucinotta, Z. Jiang, H. Wang, Y. Wang, I. Rungger, S. Sanvito, and S. Hou, "Current-induced phonon renormalization in molecular junctions," Phys. Rev. B 94, 035411 (2016).

${ }^{23}$ J.-T. Lü, M. Brandbyge, P. Hedegard, T. N. Todorov, and D. Dundas, "Current-induced atomic dynamics, instabilities, and Raman signals: Quasiclassical Langevin equation approach, Phys. Rev. B 85, 245444 (2012).

${ }^{24}$ B. Li, E. Y. Wilner, M. Thoss, E. Rabani, and W. H. Miller, "A quasi-classical mapping approach to vibrationally coupled electron transport in molecular junctions," J. Chem. Phys. 140, 104110 (2014); E. Y. Wilner, H. Wang, M. Thoss, and E. Rabani, "Sub-Ohmic to super-Ohmic crossover behavior in molecular tunneling junctions with electron-phonon interactions," Phys. Rev. B 92, 195143 (2015).

${ }^{25}$ T. M. Caspary and U. Peskin, "Electronic transport through molecular junctions with nonrigid molecule-leads coupling," J. Chem. Phys. 127, 154706 (2007).

${ }^{26} \mathrm{R}$. Jom and T. Seidman, "Competition between current-induced excitation and bath-induced decoherence in molecular junctions," J. Chem. Phys. 131, 244114 (2009).

${ }^{27}$ N. A. Zimbovskaya and M. M. Kuklja, "Vibration-induced inelastic effects in the electron transport through multisite molecular bridges," J. Chem. Phys. 131, 114703 (2009).

${ }^{28}$ U. Harbola, M. Esposito, and S. Mukamel, "Quantum master equation for electron transport through quantum dots and single molecules," Phys. Rev. B 74, 235309 (2006); R. Härtle and M. Thoss, "Resonant electron transport in single-molecule junctions: Vibrational excitation, rectification, negative differential resistance, and local cooling," ibid. 83, 115414 (2011); R. Härtle and M. Kulkarni, "Effect of broadening in the weak-coupling limit of vibrationally coupled electron transport through molecular junctions and the analogy to quantum dot circuit QED systems," ibid. 91, 245429 (2015).

${ }^{29}$ L. Mühlbacher and E. Rabani, "Real-time path integral approach to nonequilibrium many-body quantum systems," Phys. Rev. Lett. 100, 176403 (2008).

${ }^{30}$ L. V. Keldysh, "Diagram technique for nonequilibrium processes," Sov. Phys. JETP 20, 1018 (1965).

${ }^{31}$ A. Mitra, I. Aleiner, and A. J. Millis, "Phonon effects in molecular transistors: Quantal and classical treatment,” Phys. Rev. B 69, 245302 (2004). 
${ }^{32}$ R. Egger and A. O. Gogolin, "Vibration-induced correction to the current through a single molecule," Phys. Rev. B 77, 113405 (2008).

${ }^{33}$ O. Entin-Wohlman, Y. Imry, and A. Aharony, "Voltage-induced singularities in transport through molecular junctions," Phys. Rev. B 80, 035417 (2009).

${ }^{34}$ O. Entin-Wohlman, Y. Imry, and A. Aharony, "Three-terminal thermoelectric transport through a molecular junction," Phys. Rev. B 82, 115314 (2010).

${ }^{35}$ A. Ueda, O. Entin-Wohlman, and A. Aharony, "Effects of coupling to vibrational modes on the ac conductance of molecular junctions," Phys. Rev. B 83, 155438 (2011).

${ }^{36}$ O. Entin-Wohlman and A. Aharony, "Three-terminal thermoelectric transport through a molecule placed on an Aharonov-Bohm ring," Phys. Rev. B 85, 085401 (2012).

${ }^{37}$ Y. Utsumi, O. Entin-Wohlman, A. Ueda, and A. Aharony, "Full-counting statistics for molecular junctions: Fluctuation theorem and singularities," Phys. Rev. B 87, 115407 (2013).

${ }^{38}$ A. Ueda, Y. Utsumi, H. Imamura, and Y. Tokura, "Phonon-induced electronhole excitation and ac conductance in molecular junction," J. Phys. Soc. Jpn. 85, 043703 (2016).

${ }^{39}$ F. Haupt, T. Novotný, and W. Belzig, "Phonon-assisted current noise in molecular junctions," Phys. Rev. Lett. 103, 136601 (2009); "Current noise in molecular junctions: Effects of the electron-phonon interaction," Phys. Rev. B 82, 165441 (2010); T. Novotný, F. Haupt, and W. Belzig, "Nonequilibrium phonon backaction on the current noise in atomic-sized junctions," ibid. 84, 113107 (2011).

${ }^{40}$ R. Avriller and A. Levy Yeyati, "Electron-phonon interaction and full counting statistics in molecular junctions," Phys. Rev. B 80, 041309(R) (2009).

${ }^{41}$ T. L. Schmidt and A. Komnik, "Charge transfer statistics of a molecular quantum dot with a vibrational degree of freedom," Phys. Rev. B 80, 041307(R) (2009)

${ }^{42}$ D. F. Urban, R. Avriller, and A. Levy Yeyati, "Nonlinear effects of phonon fluctuations on transport through nanoscale junctions," Phys. Rev. B 82, 121414(R) (2010).

${ }^{43}$ M. Galperin, A. Nitzan, and M. A. Ratner, "Resonant inelastic tunneling in molecular junctions," Phys. Rev. B 73, 045314 (2006).

${ }^{44}$ R. Seoane Souto, A. Levy Yeyati, A. Martín-Rodero, and R. C. Monreal, "Dressed tunneling approximation for electronic transport through molecular transistors," Phys. Rev. B 89, 085412 (2014).

${ }^{45} \mathrm{~L}$. Simine and D. Segal, "Vibrational cooling, heating, and instability in molecular conducting unctions: Full counting statistics analysis," Phys. Chem. Chem. Phys. 14, 13820 (2012).

${ }^{46}$ S. Maier, T. L. Schmidt, and A. Komnik, "Charge transfer statistics of a molecular quantum dot with strong electron-phonon interaction," Phys. Rev. B 83, 085401 (2011)

${ }^{47}$ G. Schaller, T. Krause, T. Brandes, and M. Esposito, "Single-electron transistor strongly coupled to vibrations: Counting statistics and fluctuation theorem," New J. Phys. 15, 033032 (2015).

${ }^{48}$ A. Martín-Rodero, A. Levy Yeyati, F. Flores, and R. C. Monreal, "Interpolative approach for electron-electron and electron-phonon interactions: From the Kondo to the polaronic regime," Phys. Rev. B 78, 235112 (2008); R. C. Monreal and A. Martín-Rodero, "Equation of motion approach to the Anderson-Holstein Hamiltonian," ibid. 79, 115140 (2009).

${ }^{49}$ P. S. Cornaglia, D. R. Grempel, and H. Ness, "Quantum transport through a deformable molecular transistor,” Phys. Rev. B 71, 075320 (2005).

${ }^{50}$ A. Erpenbeck, R. Härtle, M. Bockstedte, and M. Thoss, "Vibrationally dependent electron-electron interactions in resonant electron transport through single-molecule junctions," Phys. Rev. B 93, 115421 (2016).

${ }^{51}$ H.-T. Chen, G. Cohen, A. J. Millis, and D. R. Reichman, "Anderson-Holstein model in two flavors of the noncrossing approximation," Phys. Rev. B 93, 174309 (2016).
${ }^{52}$ B. Kubala and F. Marquardt, "AC conductance through an interacting quantum dot," Phys. Rev. B 81, 115319 (2010).

${ }^{53}$ G.-H. Ding and B. Dong, "Phonon effects on the current noise spectra and the ac conductance of a single molecular junction,” J. Phys.: Condens. Matter 26, 305301 (2014).

${ }^{54}$ T. Holstein, "Studies of polaron motion: Part I. The molecular-crystal model," Ann. Phys. 8, 325 (1959).

${ }^{55}$ M. Galperin, M. A. Ratner, and A. Nitzan, "Molecular transport junctions: Vibrational effects," J. Phys.: Condens. Matter 19, 103201 (2007).

${ }^{56}$ Y. Utsumi, D. S. Golubev, and G. Schön, "Full counting statistics for a singleelectron transistor: Nonequilibrium effects at intermediate conductance," Phys. Rev. Lett. 96, 086803 (2006).

${ }^{57}$ O. Entin-Wohlman, Y. Imry, and A. Aharony, "Transport through molecular junctions with a nonequilibrium phonon population," Phys. Rev. B 81, 113408 (2010); A similar problem had been encountered for a local moment coupled with a nonequilibrium electron gas, see, e.g, A. Rosch, J. Paaske, J. Kroha, and P. Wölfle, "Nonequilibrium transport through a Kondo dot in a magnetic field: Perturbation theory and poor mans scaling," Phys. Rev. Lett. 90, 076804 (2003); O. Parcollet and C. Hooley, "Perturbative expansion of the magnetization in the out-of-equilibrium Kondo model," Phys. Rev. B 66, 085315 (2002).

${ }^{58}$ C. Emary, D. Marcos, R. Aguado, and T. Brandes, "Frequency-dependent counting statistics in interacting nanoscale conductors," Phys. Rev. B 76, 161404(R) (2007).

${ }^{59}$ J. Tobiska and Yu. V. Nazarov, "Inelastic interaction corrections and universal relations for full counting statistics in a quantum contact," Phys. Rev. B 72, 235328 (2005).

${ }^{60}$ H. Förster and M. Büttiker, "Fluctuation relations without microreversibility in nonlinear transport," Phys. Rev. Lett. 101, 136805 (2008).

${ }^{61} \mathrm{~K}$. Saito and Y. Utsumi, "Symmetry in full counting statistics, fluctuation theorem, and relations among nonlinear transport coefficients in the presence of a magnetic field," Phys. Rev. B 78, 115429 (2008).

${ }^{62}$ Y. Utsumi and K. Saito, "Fluctuation theorem in a quantum-dot AharonovBohm interferometer," Phys. Rev. B 79, 235311 (2009).

${ }^{63}$ D. Andrieux, P. Gaspard, T. Monnai, and S. Tasaki, "The fluctuation theorem for currents in open quantum systems," New J. Phys. 11, 043014 (2009).

${ }^{64}$ M. Esposito, U. Harbola, and S. Mukamel, "Nonequilibrium fluctuations, fluctuation theorems, and counting statistics in quantum systems," Rev. Mod. Phys. 81, 1665 (2009).

${ }^{65}$ M. Campisi, P. Hänggi, and P. Talkner, "Colloquium: Quantum fluctuation relations: Foundations and applications," Rev. Mod. Phys. 83, 771 (2011).

${ }^{66}$ A. Altland, A. De Martino, R. Egger, and B. Narozhny, "Fluctuation relations and rare realizations of transport observables," Phys. Rev. Lett. 105, 170601 (2010); "Transient fluctuation relations for time-dependent particle transport," Phys. Rev. B 82, 115323 (2010).

${ }^{67}$ R. López, J. S. Lim, and D. Snchez, "Fluctuation relations for spintronics," Phys. Rev. Lett. 108, 246603 (2012).

${ }^{68}$ Y. Utsumi, D. S. Golubev, M. Marthaler, K. Saito, T. Fujisawa, and G. Schön, "Bidirectional single-electron counting and the fluctuation theorem," Phys. Rev. B 81, 125331 (2010).

${ }^{69}$ A. G. Abanov and D. A. Ivanov, "Allowed charge transfers between coherent conductors driven by a time-dependent scatterer," Phys. Rev. Lett. 100, 086602 (2008); Phys. Rev. B 79, 205315 (2009).

${ }^{70} \mathrm{C}$. N. Yang and T. D. Lee, "Statistical theory of equations of state and phase transitions. I. Theory of condensation," Phys. Rev. 87, 404 (1952); T. D. Lee and C. N. Yang, "Statistical theory of equations of state and phase transitions. II. Lattice gas and Ising model,” Phys. Rev. 87, 410 (1952).

${ }^{71}$ C. Flindt and J. P. Garrahan, "Trajectory phase transitions, Lee-Yang zeros, and high-order cumulants in full counting statistics," Phys. Rev. Lett. 110, 050601 (2013). 